\title{
Data-Driven Sensor Clustering and Filtering for Communication Efficient Field Reconstruction
}

\author{
Jia Chen, Akshay Malhotra, and Ioannis D. Schizas ${ }^{1}$ \\ University of Texas at Arlington, Department of Electrical Engineering \\ 416 Yates Street, Arlington, TX 76010, USA
}

\begin{abstract}
A novel communication efficient scheme for reconstructing a field sensed by spatially scattered sensors is proposed. The field is formed by multiple sources, while a fusion center gathers the sensor measurements. The goal is to reconstruct the field at the fusion center using only the measurements of a small number of sensors. The framework entails learning the correlation structure of the field by determining clusters of correlated sensors observing the same set of sources. Combining moving-average filtering along with principal component analysis applied in the sensor data covariance the number of sources can be determined, while norm-one regularized canonical correlations are utilized to determine the different correlated clusters. A novel iterative interplay of regularized canonical correlations with principal component analysis is designed that determines correctly the correlated clusters as the number of training data goes to infinity. From each cluster only a head sensor transmits data to the fusion center, while the measurements of the remaining sensors are reconstructed using proper linear filters that learn the correlation pattern within a cluster via normalized least mean squares. The novel approach substantially reduces the communication cost. Extensive numerical tests demonstrate the effectiveness of the proposed scheme in field recovery.

Keywords: Field reconstruction, norm-one regularized canonical correlation analysis, clustering, adaptive filtering, communication efficiency.
\end{abstract}

Email address: jia.chen81@mavs.uta.edu, akshay.malhotra@mavs.uta.edu, schizas@uta.edu (Jia Chen, Akshay Malhotra, and Ioannis D. Schizas)

${ }^{1}$ This work is supported by the National Science Foundation under Grants CCF-1218079 and ECCS1509780

Preprint submitted to Elsevier Signal Processing

October 14, 2016

(C) 2016. This manuscript version is made available under the Elsevier user license http://www.elsevier.com/open-access/userlicense/1.0/ 


\section{Introduction}

The usage of large number of scattered sensors in environmental monitoring is gaining more and more popularity due to the low cost and simplicity of the sensing units. Sensors' main task is to monitor a field and continuously acquire spatio-temporal samena are induced by sources, such as thermal or chemical sources, causing temperature variations in a field [35], air pollution, or light intensity variations [1]]. One popular architecture when using sensing units is the fusion-center (FC) based sensor network, where the sensing units transmit their measurements to the FC. Traditionally, all sensors transmit their data to the FC which usually causes large communication costs and results in resource depletion across all sensors [177, 5]. The main focus of this work is to design a scheme where only a small number of sensors communicate their data to the FC, while the FC will reconstruct the field measurements at all other sensors using only the information acquired from properly selected sensing units.

The main task of this paper therefore is to derive an efficient centralized data fusion scheme to reconstruct the sensor measurements induced by multiple uncorrelated sources present in the field. Toward this end, a novel framework is proposed that carries out the following three tasks: i) Estimating the number of field sources; ii) Clustering the sensors based on which sources they are observing; and iii) Utilizing only the measurements of a few cluster head sensors to reconstruct all sensors' measurements at the FC. To achieve the first task, moving-average (MA) filtering [10] is combined with principal component analysis (PCA) [3] to eliminate sensing noise variance and extract the number of principal components (PCs) in the sensor data covariance corresponding to the uncorrelated sources. To group sensors in clusters according to their

source information, the main idea is to realize that sensor measurements containing information about the same sources are statistically correlated. To exploit such spatial correlations, a norm-one regularized canonical correlation analysis technique [4] will be used to extract correlated sensor measurements and group them in clusters. PCA and adaptive filtering will also be utilized to result in an improved clustering approach 
that groups sensors according to their information content. Normalized least mean squares is utilized to enable the FC to reconstruct each cluster's sensor measurements using only data from a few cluster head sensors that communicate their observations to the FC. Different from existing source-based clustering techniques [4, 6] the proposed framework is able to perform accurate clustering even when sensors observe multiple sources. The main idea is to separate the network into clusters of similar information content and use within each cluster the data of only one sensor to reconstruct the remaining sensor measurements at the FC. Such an approach will reduce significantly the number of scalars transmitted from the sensors to the FC potentially extending the sensors' lifespan.

The paper is structured as follows. Sec. 3 introduces the system setting and problem statement. Sec. 耳 outlines the basic steps involved in the novel algorithmic framework. Specifically, MA filtering is combined with PCA to determine the number of sources [Sec. 4. W]. Clustering of the sensors along with learning/reconstructing all sensor measurements is delineated in Secs. 4.2, 4.2.2 and 4.4, respectively. In Sec. 4.3 it is demonstrated that for sufficiently large number of measurements, the proposed scheme achieves flawless sensor clustering, while a simple technique is put forth to improve clustering performance for a small number of training data. Sec. \$ provides communication and computational costs at the FC. Extensive numerical tests are carried out in Sec. 6, demonstrating the potential of the proposed framework.

\section{Related Work}

Typically, sensors are limited in terms of communication and computational capabilities. A straightforward approach to collect the information across all sensors to the FC is to allow each sensor to forward its acquired measurements, possibly via multihop communications, to the FC. Such a process can place a heavy operational burden

55 in all sensors due to the high communication cost. To tackle such a challenge, data aggregation techniques have become crucial to prolong the overall lifespan of a sensor network. There are four different strategies for data aggregation: i) centralized approaches, ii) in-network aggregation, iii) tree-based approaches, and iv) cluster-based aggregation [20]. The work in [1] considers a dynamic spanning-tree approach to mini- 
mize the energy consumption by taking into consideration the data traffic load. Support vector machines are used in [21] to reduce the redundant data and eliminate false data. An efficient cluster-based data aggregation scheme for heterogeneous sensor networks was developed in [15], where inter- (intra-) cluster data aggregation is performed to eliminate redundant data. The cluster-based approach in [25] uses a context-aware approach to validate data, while intra-cluster and inter-cluster redundancy is eliminated when sensors belong to the same cluster or neighboring clusters, respectively, for the validated data. The work in [28] focuses on the issues of accuracy, traffic load, redundancy elimination and delay when performing data aggregation, and proposed a model to address the aforementioned issues.

70 In the aforementioned cluster-based approaches, the geographical area is divided into multiple grid-based clusters, while the cluster heads are elected as those sensors with the highest energy and largest number of one-hop neighboring sensors, or as the sensors whose positions are closer to the centroid position of the cluster. Further, there is no basic principle in deciding the number of clusters needed. There are fundamental differences with the work proposed here. Specifically, the sensors will be clustered in groups according to their information content and the sources they sense (possibly multiple). Thus, the clusters here are formed based on the sensor information content and not according to an ad hoc splitting of the area monitored. Further, the number of clusters will be determined by the number of the underlying information sources in the 80 monitored field. Last but not least, the clustering proposed here is done to facilitate a form of reduction in the number of data transmitted and achieve accurate reconstruction at the FC.

Clustering sensors based on their source information has been considered in [26, 4]. In [26], a distributed sparsity-aware matrix decomposition framework to estimate the support of the sparse covariance factors was proposed, which was further used in identifying source-informative sensors. A more generalized scheme for grouping sensors according to their source content was put forth in [4], which was able to deal with nonlinear data models. One challenge in the aforementioned approaches is that the number of sources is assumed to be known, while the source-to-sensor propagation channels used consider only flat fading. Another contribution of our work is the consideration 
of sensors acquiring information about multiple sources, which was not addressed effectively in [26, 4].

There are various algorithms addressing the problem of clustering data into different groups in which the data share similar properties. The most popular technique is the K-means algorithm [13, 7], which represents clusters by centroid points and allocates each data vector to the cluster that has the most similar centroid with respect to a distance metric. These clustering schemes are challenged by the fact that in our setting, sensors belonging to the same cluster do not necessarily exhibit similar magnitude in their observations, while sensors may be observing multiple sources resulting in overlapping clusters.

The field reconstruction problem has been previously addressed in the literature in [2] under the assumption that the monitored field is spatially governed by known partial differential diffusion equations. An interesting work can also be found in [23], where an optimal dimensionality-reduced approximation method was developed to recover thermal maps. Based on Bayesian estimation and Kalman filtering, the papers [118, 31, 33, 30] consider statistical estimation methods for non-static fields. Algorithms to estimate a single source's parameters are studied in [16, 9, 12] to fully recover the monitored field. The work in [119] puts forth an algorithm that can successfully reconstruct sensor signals as long as the field bandwidth is sufficiently small, the field adheres to an one-dimensional model and the sensor locations are known to the FC. A distributed cluster-based signal reconstruction for non-bandlimited fields is proposed in [24], by locally adapting the field model. In [29], a distributed adaptive node-specific signal estimation (DANSE) algorithm is considered in wireless sensor networks to estimate a set of desired signals, however, the effects of nonideal propagation channels are not considered. However, the aforementioned schemes either assume the fields are driven by assumed known spatiotemporal diffusion equations or statistical models, or only take a single-source into consideration. Different from the existing methods, our method is attractive since it does not require all sensor to communicate with the $\mathrm{FC}$, it does not require knowledge of the sensors' positions, it can address settings where the field consists of multiple spatially scattered sources and the sources-to-sensors propagation channels may be multipath. There is no need to estimate the source signals, 
and our focus is on reconstructing the field only in points of interest where sensors are deployed rather than the entire field.

\section{Problem Formulation}

125 3.1. System Model

Consider $p$ spatially scattered sensors. The sensors monitor a field which is generated by $M$ underlying sources, while the number of sources $M$ is not available. The sources are placed in different spatial locations, e.g., thermal sources, while the source signals are modeled as random uncorrelated processes, namely $s_{m}(t)$, where $m$ is the source index and $t$ denotes time. The source signals are assumed to be wide sense stationary, which implies that their ensemble average is time-invariant.

The field sources' signals are reaching the sensing units via multipath propagation channels. The channel coefficients from the $m$ th source to sensor $j$ is modeled as a finite impulse response filter with coefficients $\mathbf{h}_{j, m}=\left[h_{j, m}(0), \ldots, h_{j, m}(L-1)\right]$, where $L$ corresponds to the maximum number of taps these filters can have. The channel coefficients are not known and modeled as random Gaussian variables. The energy of the source signals is assumed to be decreasing exponentially with distance (moving away from the source), which is similar to the propagation mechanics of a field in [2]. Motivated by the data model in [22, [36], the measurements acquired at sensor $j$ and time $t$ adhere to the linear superposition model

$$
x_{j}(t)=\sum_{m \in \mathcal{S}_{j}} c_{j, m} \sum_{\tau=0}^{L-1} h_{j, m}(\tau) s_{m}(t-\tau)+w_{j}(t)
$$

where, $\mathcal{S}_{j}$ contains the indices of the sources observed by sensor $j, w_{j}(t)$ corresponds to the zero-mean sensing noise with variance $\sigma_{w, j}^{2}$, and $c_{j, m}$ is a positive coefficient quantifying the attenuation experienced by the $m$ th source signal at sensor $j$. Given that the source signal power attenuates exponentially fast with the propagation distance, the attenuation coefficients are written as

$$
c_{j, m}=\frac{1}{4 \pi} e^{-\left\|\mathbf{p}_{j}-\mathbf{p}_{s_{m}}\right\|^{2}}
$$

where $\mathbf{p}_{j}, \mathbf{p}_{s_{m}} \in \mathbb{R}^{2}$ correspond to the positions of sensor $j$ and source $s_{m}(t)$, respectively, and $\|\cdot\|_{2}$ denotes Euclidean norm. 


\subsection{Problem Description}

In existing sensing protocols developed for FC-based topologies, all sensor mea-

150 surements at any time instant $t$ have to be transmitted back to the FC for application dependent processing. Thus, at every $t$ all $p$ sensors transmit to the FC, leading to a communication cost of the order of $\mathcal{O}(p t)$. The communication cost is prohibitively high given that the number of sensors $p$ is large for many different sensing applications such as environmental monitoring, surveillance and so on [8, [34].

The objective here is to utilize the spatial correlation among the sensor measurements to significantly reduce the communication load. The aforementioned goal can be divided into two steps: i) Identifying the set of sensors acquiring spatially correlated measurements; and ii) Learning the statistical models that the different correlated groups of sensor measurements follow. Toward this end, the first step boils down to determining the sets $\mathcal{M}_{m}, \forall m \in\{1, \ldots, M\}$ that contain the sensor indices whose measurements contain information about source $s_{m}(t)$.

The second step relies on the fact that a set of correlated sensor measurements are linearly dependent on common source signals. The main idea is to transmit the measurements of only one sensor in a correlated set $\mathcal{M}_{m}$, namely a head sensor, and rely on adaptive filtering to learn proper linear transformations (filtering coefficients) that can be used to reconstruct the data of all other sensors in $\mathcal{M}_{m}$ using only the head sensor's measurements. Learning has to be performed under a setting where the sourceto-sensor channel coefficients are unknown, and source signals are not available. In detail, in every set of correlated measurements $\mathcal{M}_{m}$, the head sensor will be designated as a reference sensor whose measurements will be used to linearly reconstruct all other sensors' measurements in $\mathcal{M}_{m}$. Thus, communication savings will be introduced by communicating to the FC only the head sensor measurements.

\section{Learning and Reconstructing the Monitored Field}

The proposed framework entails a training phase during which training data are acquired at the sensors and used to learn the statistical correlation structure of the monitored field. Specifically, three tasks are carried out here: i) determining the unknown 
number of sources $M$; ii) identifying the $M$ sets of sensors with correlated measurements, i.e., $\left\{\mathcal{M}_{m}\right\}_{m=1}^{M}$; and iii) learning pertinent filters to reconstruct the sensor measurements in a correlated set $\mathcal{M}_{m}$ using only the head sensor's acquired measurements.

\subsection{Determining the Number of Sources}

During training each sensor, say sensor $j$, acquires $N$ measurements $x_{j}(t)$ for $t=$ $1, \ldots, N$ adhering to the model in (II). Each sensor then performs sample-averaging using a moving-average (MA) filter, producing the MA processed measurements

$$
\bar{x}_{j}(t)=P^{-1} \sum_{\ell=1}^{P} x_{j}(t+\ell-1)
$$

where $P$ denotes the length of the MA filter performing averaging, with $t=1, \ldots, N-$

185

190 $P+1$, while $N>P$.

The next step is to stack all the MA measurements in (B]) in a vector $\overline{\mathbf{x}}_{t}:=\left[\bar{x}_{1}(t)\right.$ $\left.\ldots \bar{x}_{p}(t)\right]^{T}$ that can be formed at the FC, where $p$ denotes the number of sensors. The MA data vectors are subsequently used to estimate the MA data covariance matrix via sample-averaging as

$$
\hat{\boldsymbol{\Sigma}}_{\bar{x}}=(N-P+1)^{-1} \sum_{t=1}^{N-P+1}\left[\overline{\mathbf{x}}_{t}-\mathbf{m}_{\bar{x}}\right]\left[\overline{\mathbf{x}}_{t}-\mathbf{m}_{\bar{x}}\right]^{T},
$$

where $\mathbf{m}_{\bar{x}}:=(N-P+1)^{-1} \sum_{t=1}^{N-P+1} \overline{\mathbf{x}}_{t}$ denotes the sample-average estimate for the mean of $\overline{\mathbf{x}}_{t}$.

It is demonstrated in Apdx. A that as the number of training data increases, while the length of the MA filter $P$ is sufficiently large to make the sensing noise variance sufficiently small (arbitrarily close to zero for increasing $P$ ), then the number of eigenvalues of $\hat{\boldsymbol{\Sigma}}_{\bar{x}}$, whose amplitude remains almost unchanged before and after MA filtering, will be equal to the number of sources $M$. Thus, the number of sources can be easily identified by observing which eigenvalues are significantly reduced after perfoming MA filtering. Effectively the MA filtering helps in reducing the sensing noise variance while preserving the source signal power as shown in Apdx. A. Moreover, MA filtering transforms the convolutive model in (II) into a simpler low dimensional linear model on which MA data $\overline{\mathbf{x}}_{t}$ adhere to. Then, PCA is utilized to determine the source-related principal eigenvalues. 


\subsection{Clustering Sensor Measurements}

The first step in the proposed field reconstruction scheme is to determine clusters sors observe more than one sources (overlapping sources). This is to be contrasted with the approach in [4] which may not perform well for an overlapping setting, where the clustering performance is sensitive to certain parameter selection.

\subsubsection{Norm-One Regularized Canonical Correlations}

A brief overview of the sparsity-aware CCA (S-CCA) clustering scheme in [4] is given here for completeness. Given the MA sensor measurements $\overline{\mathbf{x}}_{t}$ for $t=1, \ldots, \bar{N}:=$ $N-P+1$, the following two data sequences are formed, namely $\left\{\chi_{t}, \boldsymbol{\psi}_{t}\right\} \in \mathbb{R}^{p f \times 1}$

$$
\chi_{t}=\left[\overline{\mathbf{x}}_{t-1}^{T}, \cdots, \overline{\mathbf{x}}_{t-f}^{T}\right]^{T}, \boldsymbol{\psi}_{t}=\left[\overline{\mathbf{x}}_{t}^{T}, \cdots, \overline{\mathbf{x}}_{t+f-1}^{T}\right]^{T}
$$

which are utilized by the S-CCA framework in the following norm-one regularized formulation $(\hat{\mathbf{D}}, \hat{\mathbf{E}})=\arg \min _{\mathbf{D}, \mathbf{E}} \bar{N}^{-1} \sum_{t=1}^{\bar{N}}\left\|\mathbf{E}\left(\boldsymbol{\psi}_{t}-\mathbf{m}_{\psi}\right)-\mathbf{D}\left(\boldsymbol{\chi}_{t}-\mathbf{m}_{\chi}\right)\right\|_{2}^{2}+\sum_{\rho=1}^{M} \lambda_{E, \rho}\left\|\mathbf{E}_{\rho:}^{T}\right\|_{1}$ $+\sum_{\rho=1}^{M} \lambda_{D, \rho}\left\|\mathbf{D}_{\rho:}^{T}\right\|_{1}+v\left\|\mathbf{E} \hat{\boldsymbol{\Sigma}}_{\psi} \mathbf{E}^{T}-\mathbf{I}\right\|_{F}^{2}+\varepsilon\left\|\mathbf{D} \hat{\boldsymbol{\Sigma}}_{\chi} \mathbf{D}^{T}-\mathbf{I}\right\|_{F}^{2}$

where $\mathbf{m}_{\psi}$ and $\mathbf{m}_{\chi}$ are the sample-averaging estimates for the mean of the sequences $\boldsymbol{\psi}_{t}$ and $\chi_{t}$, respectively, while $\hat{\boldsymbol{\Sigma}}_{\psi}$ and $\hat{\boldsymbol{\Sigma}}_{\chi}$ denote the corresponding sample-averaging covariance estimates, and $\mathbf{I} \in \mathbb{R}^{M \times M}$ denotes the identity matrix. Furthermore, the

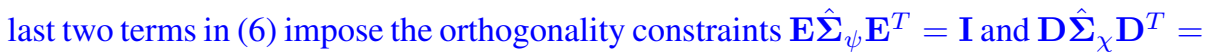
I present in the standard CCA and which are needed to recover the different uncorrelated source signals $\left\{s_{m}(t)\right\}_{m=1}^{M}$, with $\varepsilon>0$ and $v>0$. Note that, $\boldsymbol{\chi}_{t}$ represents the past sensor measurements with respect to (w.r.t.) time instant $t$, and $\boldsymbol{\psi}_{t}$ spans the present and future of sensor data sequence $\overline{\mathbf{x}}_{t}$. Further, the positive integer $f$ represents 
memory length. S-CCA different from standard CCA is trying to recover sparse matrices $\mathbf{D}, \mathbf{E} \in \mathbb{R}^{M \times p f}$ with $M \leq p f$. The support (nonzero entries) of each of the sparse rows in $\mathbf{E}$ and $\mathbf{D}$ will indicate the set of sensors observing the same source carrying out in that way the aforementioned clustering task (see details in [4]). For example, consider a field in which $M=2$ uncorrelated sources are present and $p=10$ sensors deployed. Sensors $\{1,2,3,4,5\}$ observe source $s_{1}(t)$ and sensors $\{6,7,8,9,10\}$ observe source $s_{2}(t)$. Then, ideally sparsity should be induced in matrices $\mathbf{D} \in \mathbb{R}^{2 \times 10}$ and $\mathbf{E} \in \mathbb{R}^{2 \times 10}$ such that one row of $\mathbf{D}$ and $\mathbf{E}$ only has nonzero entries in indices $\{1,2,3,4,5\}$ and another row only has nonzero entries in indices $\{6,7,8,9,10\}$. Notice that, matrices $\mathbf{D}$ and $\mathbf{E}$ have the same (non)zero support (not necessarily have the same value), thus, the clustering task can be carried out by checking the support of either $\mathbf{D}$ or $\mathbf{E}$.

The positive sparsity-controlling coefficients $\lambda_{E, \rho}, \lambda_{D, \rho}$ control the number of (non)zero entries in the $\rho$ th row of $\mathbf{E}, \mathbf{D}$. Proper selection of coefficients $\lambda_{E, \rho}, \lambda_{D, \rho}$ can induce proper sparsity in the rows of the matrices $\hat{\mathbf{D}}$ and $\hat{\mathbf{E}}$, where the nonzero entries indices can point to those sensors acquiring information about the same source. S-CCA as demonstrated in [4] can perform clustering correctly with high probability.

The minimization problem in (G) is nonconvex w.r.t. $\mathbf{D}$ and $\mathbf{E}$. In order to come around this obstacle block coordinate descent (BCD) techniques will be applied. To facilitate application of BCD during coordinate cycle $\tau$ the cost in (G) is rewritten as

$$
\begin{aligned}
& \left(\hat{\mathbf{D}}^{\tau}, \hat{\mathbf{E}}^{\tau}\right)=\arg \min _{\mathbf{D}, \mathbf{E}} \bar{N}^{-1} \sum_{t=1}^{\bar{N}}\left\|\mathbf{E} \boldsymbol{\psi}_{t}-\mathbf{D} \boldsymbol{\chi}_{t}-\mathbf{m}\right\|_{2}^{2}+\sum_{\rho=1}^{M} \lambda_{E, \rho}\left\|\mathbf{E}_{\rho:}^{T}\right\|_{1} \\
& +\sum_{\rho=1}^{M} \lambda_{D, \rho}\left\|\mathbf{D}_{\rho}^{T}\right\|\left\|_{1}+v\right\| \mathbf{E} \hat{\boldsymbol{\Sigma}}_{\psi}\left(\hat{\mathbf{E}}^{\tau-1}\right)^{T}-\mathbf{I}\left\|_{F}^{2}+\varepsilon\right\| \mathbf{D} \hat{\boldsymbol{\Sigma}}_{\chi}\left(\hat{\mathbf{D}}^{\tau-1}\right)^{T}-\mathbf{I} \|_{F}^{2}
\end{aligned}
$$

where $\mathbf{m}:=\mathbf{E m}_{\psi}-\mathbf{D m}_{\chi}$. Notice that the last two terms in (G) are substituted by $v\left\|\mathbf{E} \hat{\boldsymbol{\Sigma}}_{\psi}\left(\hat{\mathbf{E}}^{\tau-1}\right)^{T}-\mathbf{I}\right\|_{F}^{2}+\varepsilon\left\|\mathbf{D} \hat{\boldsymbol{\Sigma}}_{\chi}\left(\hat{\mathbf{D}}^{\tau-1}\right)^{T}-\mathbf{I}\right\|_{F}^{2}$ in (पD), in order to avoid fourthorder polynomial terms, where $\hat{\mathbf{D}}^{\tau-1}$ and $\hat{\mathbf{E}}^{\tau-1}$ represent the estimates of $\mathbf{D}$ and $\mathbf{E}$ during the $(\tau-1)$ th coordinate cycle. The cost in (पI) is minimized w.r.t. one entry of $\mathbf{D}$ (or $\mathbf{E}$ ), while fixing the remaining entries of $\mathbf{D}$ (or $\mathbf{E}$ ) to their most up-to-date

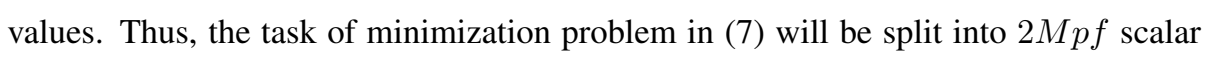
minimization subproblems, corresponding to each of the entries of the matrix $\mathbf{D}$ or E. The scalar update of each entry, i.e., $\hat{\mathbf{D}}^{\tau}(\alpha, \beta)$, can be obtained by minimizing the 
following problem

$$
\begin{aligned}
& \hat{\mathbf{D}}^{\tau}(\alpha, \beta)=\arg \min _{d}\left\|\boldsymbol{\psi}_{\alpha, \beta}^{\tau}-d \mathbf{h}_{\alpha, \beta}^{\tau}\right\|_{2}^{2}+\lambda_{D, \alpha}|d|+\left\|\breve{\boldsymbol{\psi}}_{\alpha, \beta}^{\tau}-d \breve{\mathbf{h}}_{\alpha, \beta}^{\tau}\right\|_{2}^{2}, \\
& \quad \text { for } \alpha=1, \ldots, M, \beta=1, \ldots, p f \text {,in which } \\
& \boldsymbol{\psi}_{\alpha, \beta}^{\tau}:=\bar{N}^{-0.5}\left(\left[\hat{\mathbf{E}}^{\tau-1} \mathbf{\Psi}\right]_{\alpha:}-\sum_{\ell=1}^{\beta-1} \hat{\mathbf{D}}^{\tau}(\alpha, \ell) \mathbf{X}_{\ell:}-\sum_{\ell=\beta+1}^{p f} \hat{\mathbf{D}}^{\tau-1}(\alpha, \ell) \mathbf{X}_{\ell:}\right) \\
& \text { and, } \mathbf{h}_{\alpha, \beta}^{\tau}:=\bar{N}^{-0.5}\left(\mathbf{X}_{\beta:}\right) \\
& \breve{\boldsymbol{\psi}}_{\alpha, \beta}^{\tau}:=\varepsilon^{0.5}\left(\mathbf{I}_{\alpha:}-\sum_{\ell=1}^{\beta-1} \hat{\mathbf{D}}^{\tau}(\alpha, \ell)\left[\hat{\boldsymbol{\Sigma}}_{\chi}\left(\hat{\mathbf{D}}^{\tau-1}\right)^{T}\right]_{\ell:}-\sum_{\ell=\beta+1}^{p f}\right. \\
& \left.\hat{\mathbf{D}}^{\tau-1}(\alpha, \ell)\left[\hat{\boldsymbol{\Sigma}}_{\chi}\left(\hat{\mathbf{D}}^{\tau-1}\right)^{T}\right]_{\ell:}\right), \breve{\mathbf{h}}_{\alpha, \beta}^{\tau}:=\varepsilon^{0.5}\left[\hat{\boldsymbol{\Sigma}}_{\chi}\left(\hat{\mathbf{D}}^{\tau-1}\right)^{T}\right]_{\beta:},
\end{aligned}
$$

where $\mathbf{X}:=\left[\chi_{1}-\mathbf{m}_{\chi}, \ldots, \chi_{\bar{N}}-\mathbf{m}_{\chi}\right]$, while $\boldsymbol{\Psi}:=\left[\boldsymbol{\psi}_{1}-\mathbf{m}_{\psi}, \ldots, \boldsymbol{\psi}_{\bar{N}}-\mathbf{m}_{\psi}\right]$, while $\mathbf{M}_{\alpha:}$ (or $[\mathbf{M}]_{\alpha:}$ ) and $\mathbf{M}_{: \ell}$ correspond to the $\alpha$ th row and $\ell$ th column of matrix $\mathbf{M}$, respectively. The entries of $\hat{\mathbf{D}}$ are updated using Gauss-Seidel style which utilizes the most-up-to-date iterates. Another way pertains to the Jacobi style which utilizes the iterates from the previous cycle and it is easier to parallelize. The reason for selecting Gauss-Seidel style is the need for less number of updating iterations to achieve the same accuracy. Formulation (ㅈ) can be solved as (see Lemma 1 in [27])

$$
\begin{gathered}
\hat{\mathbf{D}}^{\tau}(\alpha, \beta)=\mathbb{F}\left(\left[\boldsymbol{\psi}_{\alpha, \beta}^{\tau}, \breve{\boldsymbol{\psi}}_{\alpha, \beta}^{\tau}\right]^{T},\left[\mathbf{h}_{\alpha, \beta}^{\tau}, \breve{\mathbf{h}}_{\alpha, \beta}^{\tau}\right]^{T}, \lambda_{D, \alpha}\right), \text { where } \\
\mathbb{F}\left(\mathbf{p}_{1}, \mathbf{p}_{2}, \lambda\right):=\operatorname{sgn}\left(\mathbf{p}_{1}^{T} \mathbf{p}_{2}+p_{3}\right) \times\left(\max \left(0,\left(\left|\frac{\mathbf{p}_{1}^{T} \mathbf{p}_{2}}{\left\|\mathbf{p}_{2}\right\|_{2}^{2}}\right|-\left(\frac{\lambda}{2\left(\left\|\mathbf{p}_{2}\right\|_{2}^{2}\right)}\right)\right)\right)\right.
\end{gathered}
$$

The updates $\hat{\mathbf{E}}^{\tau}(\alpha, \beta)$ can be obtained in a similar fashion after fixing the remaining entries of matrix $\mathbf{E}$ to their most recent updates, and set $\mathbf{D}$ to $\hat{\mathbf{D}}^{\tau-1}$ in (पD). In summary, the S-CCA algorithm can be summarized in Alg. 1

The clustering performance of S-CCA is affected by the selection of the sparsitycontrolling coefficients $\lambda_{E, \rho}$ and $\lambda_{D, \rho}$. An effective yet simple approach to select these coefficients can be found in [4, Sec. III. C ], and the process of applying SCCA combined with the regularization coefficient selection method is given in Alg. 2. Nonetheless, the clustering performance of S-CCA may not be perfect. To improve performance, an iterative combination of the S-CCA framework and PCA framework is proposed next. As it will be shown in Sec. 4.3 this combination can lead to perfect sensor clustering as the number of measurements $N$, and MA filter length $P$ are increasing. 


\subsubsection{Iterative Cluster Splitting and Merging}

The idea here is to determine how many sources are present in each of the estimated clusters $\hat{\mathcal{M}}_{m}$ obtained from S-CCA when applied in all data. If a cluster has one source no further splitting is needed, whereas if a cluster contains information about multiple sources the goal is to identify which sources are contained in that cluster. For each of the clusters $\hat{\mathcal{M}}_{m}$ the corresponding sensor measurements are stacked in vectors $\overline{\mathbf{x}}_{m^{1}}^{1}(t)$, where the subscript indicates the cluster index and the superscript the iteration index of the alternating process between S-CCA and PCA, while $t=1, \ldots, \bar{N}$ and $m^{1} \equiv m=1, \ldots, M$. These vectors are used to form the sample-averaging covariance estimates

$$
\boldsymbol{\Sigma}_{x, m^{1}}^{1}:=\bar{N}^{-1} \sum_{\tau=1}^{\bar{N}}\left[\overline{\mathbf{x}}_{m^{1}}^{1}(\tau)-\mathbf{m}_{\bar{x}_{m^{1}}^{1}}\right]\left[\overline{\mathbf{x}}_{m^{1}}^{1}(\tau)-\mathbf{m}_{\bar{x}_{m^{1}}^{1}}\right]^{T},
$$

where $\mathbf{m}_{\bar{x}_{m^{1}}^{1}}$ denotes the sample-average of vectors $\left\{\overline{\mathbf{x}}_{m^{1}}^{1}(t)\right\}_{t=1}^{\bar{N}}$. Here PCA can be applied to determine the number of source-related PCs, namely $M_{m^{1}}$ for $m^{1}=$ $1, \ldots, \hat{M}$ where $\hat{M}$ is the estimate of $M$ using the method described in Sec. 4.J. For sufficiently large $P$ (length of MA filter) the noise-related eigenvalues in (1L2) will be significantly smaller than the source-related PCs which can be easily separated using thresholding. $M_{m^{1}}$ can be used to estimate the number of sources for which information is contained in each cluster $\hat{\mathcal{M}}_{m^{1}}$.

For each cluster $\hat{\mathcal{M}}_{m^{1}}$ for which $M_{m^{1}}=1$ no further splitting is applied in the cluster since it has been estimated that only one source is present in the measurements in $\hat{\mathcal{M}}_{m^{1}}$. However, if $M_{m^{1}}>1$ this implies that more than one sources are sensed by the measurements in $\hat{\mathcal{M}}_{m^{1}}$, and S-CCA is applied within the cluster in order to separate the measurements further based on their information content. Toward this end, the S-CCA formulation in (पD) can be applied after using matrices $\mathbf{E}$ and $\mathbf{D}$ of size $M_{m^{1}} \times\left|\hat{\mathcal{M}}_{m^{1}}\right| f$ where $\left|\hat{\mathcal{M}}_{m^{1}}\right|$ denotes the number of measurements in $\hat{\mathcal{M}}_{m^{1}}$. Further, the $\chi_{t}$ and $\boldsymbol{\psi}_{t}$ vectors will be of size $\left|\hat{\mathcal{M}}_{m^{1}}\right| f \times 1$ and constructed as described earlier using only the measurements in $\hat{\mathcal{M}}_{m^{1}}$. Thus, if $M_{m^{1}}>1$ S-CCA is employed (iteration index 2) to perform further splitting of cluster $\hat{\mathcal{M}}_{m^{1}}$ into $M_{m^{1}}$ clusters denoted as $\hat{\mathcal{M}}_{m^{1}, m^{2}}$, where $m^{2}=1, \ldots, M_{m^{1}}$ and $m^{1} \in\{1, \ldots, \hat{M}\}$ for all these clusters that give more than one PCs after iteration 2. Then, PCA is applied again during iteration 3 to decide whether any of the clusters $\hat{\mathcal{M}}_{m^{1}, m^{2}}$ need to be split further as described 
earlier. The aforementioned iterative alternation between S-CCA and PCA is continued until all resulting clusters contain measurements whose corresponding covariance contains at most one source-related PC, or the resulting cluster contains a single measurement which may have information about multiple sources.

Next, PCA is applied to (i) merge clusters that contain information about the same source; and (ii) further conclude which sources are contained in the single-measurement clusters which sense multiple sources. To carry out task (i) any two clusters whose covariance has a single source-related eigenvalue are combined into a single-cluster if the resulting cluster gives a measurement covariance matrix which also has a single sourcerelated eigenvalue. PCA is used again to find the source-related PCs. If only one PC is present in the covariance of the resulting cluster then it is ensured that both clusters combined contain information about the same source and merging is valid, otherwise merging is not valid. Task (ii) focuses on identifying which single-source clusters carry information about the sources contained in these single-measurement clusters that sense more than one sources. After completion of task (i) each of the remaining single-measurement clusters are merged with those single-source clusters that contain measurements of sensors that are physical neighbors. This is done since sources sensed by the same sensor have to be geographically close in distance. In the merging of the neighboring clusters with a single-measurement cluster, we select only those neighboring clusters that, when merged with the single-measurement cluster, do not generate a covariance matrix with a number of PCs greater than the number of single-source neighboring clusters (which actually is equal to the number of sources contained in the single-measurement cluster). This process ensures that clustering is performed correctly for a sufficiently large number of measurements and points to the single-source clusters whose source content is contained in the examined single-measurement cluster (see details in Prop. 1).

\subsection{Algorithmic Analysis and Practical Considerations}

Interestingly, as the number of training data $N$ and the length of the MA filter $P$ are increasing arbitrarily large, alternating applicability of the S-CCA and the PCA can achieve flawless clustering of the sensors according to their source content. Specifi- 
cally, it is demonstrated in Apdx. B: in Secs. 4.2.] and 4.2.2, performs correct clustering of the sensors with probability one as the number of data $N$ and the length of the MA filter $P$ are increasing to infinity.

In practice both $N$ and $P$ are finite, which may create challenges when determining the source-related PCs and trying to separate them from the noise-related PCs when applying PCA to split or merge clusters. Specifically, in a scenario where there are sensors whose measurements contain information about multiple sources, S-CCA may result in clusters where some measurements contain information about a single source, while other measurements contain many sources. When $N$ and $P$ are sufficiently large applying PCA as delineated earlier will result in multiple source-related PCs corresponding to the sources contained in a cluster. However, when $N$ and $P$ are small due to e.g., low sampling rates, it may be the case that some source-related PCs have small-amplitude and cannot be distinguished from noise-related PCs, especially if some sources have weak presence in the cluster measurements. A process is described next to separate within a cluster sensors that contain information about one source (single-source sensors), from sensors whose measurements are affected by multiple sources (multi-source sensors).

Within a cluster, say $\hat{\mathcal{M}}$, that potentially contains both single-source and multisource sensors the goal is to extract out the single-source sensors and place them in the correct single-source cluster. To this end, the process starts by picking a sensor's measurements inside a cluster as a reference signal and checking its ability to reconstruct (via linear filtering) another sensor's measurements within the cluster. Proper linear filters can be determined via the normalized least mean squares (NLMS) process detailed in Sec. 4.4. If the reference sensor picked within a cluster is a single-source sensor and there are other single-source sensors within the cluster observing the same source, then the NLMS reconstruction error will have a variance relatively close to the sensing noise variance $\sigma_{w}^{2}$, see e.g., [32]. Let us denote the subset that contains all these single-source sensors whose measurements can be reconstructed from the reference sensor as $\mathcal{N}_{s}$. This is to be contradicted with the case where either i) the reference 
sensor picked is a multi-source sensor, or ii) the remaining sensors within the cluster are multi-source sensors. If all reconstruction errors between the reference sensor and the rest of the sensors in the cluster have variance larger than $\sigma_{w}^{2}$, it can be concluded that the single-source set $\mathcal{N}_{s}$ will be empty.

If the resulting set $\mathcal{N}_{s}$ of potential single-source sensors is not empty, then these are removed from cluster $\hat{\mathcal{M}}$ and merged with that single-source cluster that contains information about the same source. This is found by employing PCA and checking which neighboring single-source cluster can be merged with the measurements from $\mathcal{N}_{s}$ without increasing the number of PCs in the resulting merged cluster. If set $\mathcal{N}_{s}$ is empty one possible scenario is that the reference sensor is single-source and the remaining measurements within the cluster examined correspond to multi-source sensors. To check such a case, the measurements corresponding to the reference sensor from cluster $\hat{\mathcal{M}}$ are merged with existing single-source clusters. If there is a single-source cluster, say $\hat{\mathcal{M}}^{\prime}$ that when merged with the measurements of the reference sensor does not result in increasing the number of PCs, then the reference sensor measurements are removed from cluster $\hat{\mathcal{M}}$ and merged with the single-source cluster $\hat{\mathcal{M}}^{\prime}$. Then, among the remaining sensors in $\hat{\mathcal{M}}$ another one is picked as a reference and the process is repeated until all remaining sensors in $\hat{\mathcal{M}}$ have been used as reference sensors.

The aforementioned process is used to extract the single-source measurements from a cluster $\hat{\mathcal{M}}$ and merge them with those single-source clusters that have the same source information content. The remaining measurements will correspond to multi-source sensors and these multi-source sensors will be merged as explained in Sec. 4.2 .2 with neighboring single-source clusters to identify their source content. The algorithm involving alternation between S-CCA and PCA is summarized as Alg. 3.

\subsection{Learning Statistical Models}

\subsubsection{Single-source clusters}

Consider a cluster $\hat{\mathcal{M}}_{m}$ obtained via the process in Secs. 4.2 .1 and 14.2 .2 and designated as a single-source cluster. Then, a head sensor $i_{m}$ is designated in cluster $\hat{\mathcal{M}}_{m}$. Since all sensors in $\hat{\mathcal{M}}_{m}$ have correlated measurements, the goal here is to learn $\left|\hat{\mathcal{M}}_{m}\right|-1$ linear filters that are able to generate all sensors' measurements in $\hat{\mathcal{M}}_{m}$ 
using as input the measurements acquired at the head sensor $i_{m}$ for $m=1, \ldots, M$. cients of the $\left|\hat{\mathcal{M}}_{m}\right|-1$ linear filters. During the operational stage only the cluster head sensors transmit their measurements to the FC which can then reconstruct the other sensors' measurements in each cluster using only the head sensor data and the learnt filter coefficients. This process will reduce significantly the communication costs.

The head sensor $i_{m}$ in each cluster $\hat{\mathcal{M}}_{m}$ is selected as the one that has the highest SNR-like quantity that can be found at sensor $j$ as $\mathrm{SNR}=\frac{\mathbb{E}_{\bar{x}_{j}(t)}}{\left|\mathbb{E}_{\bar{x}_{j}(t)}-\mathbb{E}_{x_{j}(t)}\right|}$, where $\mathbb{E}_{\bar{x}_{j}(t)}$ is the energy of the moving-average filter output and $\left|\mathbb{E}_{\bar{x}_{j}(t)}-\mathbb{E}_{x_{j}(t)}\right|$ is an estimate of the sensing noise variance present in the sensor $j$. The head sensor will be the one which achieves the maximum SNR in a cluster. During the training phase of the algorithm all sensors in $\hat{\mathcal{M}}_{m}$ send their measurements to the FC. The FC treats the head sensor measurements $x_{i_{m}}(t)$ as input, and each of the remaining $\left|\hat{\mathcal{M}}_{m}\right|-1$ sensor measurements in $\hat{\mathcal{M}}_{m}$ is viewed as desired output of a linear filter whose coefficients are determined via the NLMS adaptive filtering approach, see e.g., [32]. Each filter is set to have $\bar{L}$ taps, where $\bar{L}$ is selected sufficiently large such that $L \ll \bar{L}$.

Consider sensor $j$ within cluster $\hat{\mathcal{M}}_{m}$ with $j \neq i_{m}$ (not the head sensor). Sensors $j$ and $i_{m}$ belong to cluster $\hat{\mathcal{M}}_{m}$, thus they should be sensing a common source $s_{m}(t)$. Taking into account the data model in $(\mathbb{I})$ and ignoring for now the sensing noise the measurements in the frequency domain are given as

$$
\mathbf{X}_{j}(\omega)=c_{j, m} \mathbf{H}_{j, m}(\omega) \mathbf{S}_{m}(\omega), \mathbf{X}_{i_{m}}(\omega)=c_{i_{m}, m} \mathbf{H}_{i_{m}, m}(\omega) \mathbf{S}_{m}(\omega)
$$

where $\omega \in[-\pi, \pi]$ denotes frequency, while $\mathbf{H}_{j, m}(\omega), \mathbf{H}_{i_{m}, m}(\omega)$ and $\mathbf{S}_{m}(\omega)$ denote the frequency responses of the source-to-sensor channels $\left\{h_{j, m}(\tau), h_{i_{m}, m}(\tau)\right\}_{\tau=0}^{L-1}$, and source $s_{m}(t)$, respectively. From (14), (15) it follows readily

$$
\mathbf{X}_{j}(\omega)=\frac{c_{j, m}}{c_{i_{m}, m}} \cdot \frac{\mathbf{H}_{j, m}(\omega)}{\mathbf{H}_{i_{m}, m}(\omega)} \cdot \mathbf{X}_{i_{m}}(\omega)
$$

Thus, the NLMS algorithm is trying to learn in time-domain (via a linear filter), the frequency response $\frac{c_{j, m}}{c_{i_{m}, m}} \cdot \frac{\mathbf{H}_{j, m}(\omega)}{\mathbf{H}_{i_{m}, m}(\omega)}$ associating the measurements at head sensor $i_{m}$, with the measurements of sensor $j$ within single-source cluster $\hat{\mathcal{M}}_{m}$. Learning the filter in (펴), via which the measurements of sensor sensor $j$ can be reconstructed using as 
input the head sensor's measurements, involves the following three steps

Step 1: Evaluate the estimated output signal for sensor $j$

$$
\hat{x}_{j}(t):=\mathbf{u}_{i_{m}}^{T}(t) \cdot \hat{\mathbf{w}}_{i_{m}, j}(t)
$$

where $\mathbf{u}_{i_{m}}^{T}(t):=\left[x_{i_{m}}(t), x_{i_{m}}(t-1), \cdots, x_{i_{m}}(t-\bar{L}+1)\right]^{T}$ is the filter input vector, $\hat{\mathbf{w}}_{i_{m}, j}(t):=\left[w_{i_{m}, j, t}(0), w_{i_{m}, j, t}(1), \cdots, w_{i_{m}, j, t}(\bar{L}-1)\right]^{T}$ contains the filter coefficients used to learn the relationship in (II4).

Step 2: Calculate the error signal $e_{i_{m}, j}(t)$

$$
e_{i_{m}, j}(t)=\hat{x}_{j}(t)-x_{j}(t)
$$

Step 3: Update the filter coefficients $\hat{\mathbf{w}}_{i_{m}, j}(t)$ as

$$
\hat{\mathbf{w}}_{i_{m}, j}(t+1)=\hat{\mathbf{w}}_{i_{m}, j}(t)+\mu \frac{e_{i_{m}, j}(t)}{\zeta+\mathbf{u}_{i_{m}}^{T}(t) \mathbf{u}_{i_{m}}(t)} \mathbf{u}_{i_{m}}(t),
$$

where $\mu$ is the step-size used in NLMS, and $\zeta$ is a positive constant. The coefficients are continuously updated at the FC until $\left\|\hat{\mathbf{w}}_{i_{m}, j}(t+1)-\hat{\mathbf{w}}_{i_{m}, j}(t)\right\|_{2}$ stops decreasing below a desired threshold. After NLMS algorithm has terminated the final filter coefficients will be denoted by $\hat{\mathbf{w}}_{i_{m}, j}$.

\subsubsection{Multi-source clusters}

When the cluster $\hat{\mathcal{M}}_{m}$ considered contains multi-source sensors some generalizations need to be performed to the aforementioned NLMS framework based adaptive filtering approach in order to reconstruct accurately the measurements of the multisource sensors within $\hat{\mathcal{M}}_{m}$. Let's denote a multi-source sensor $j$ in $\hat{\mathcal{M}}_{m}$, for each source $n \in \mathcal{S}_{j}$ a head sensor, namely $i_{n}$, is picked from a neighboring single-source cluster $\hat{\mathcal{M}}_{n}$ that contains information about source $n$. Since sensor $j$ observes all sources in set $\mathcal{S}_{j}$ the corresponding frequency domain equations of (14), (15) are written here as

$$
\begin{aligned}
& \mathbf{X}_{j}(\omega)=\sum_{n \in \mathcal{S}_{j}} c_{j, n} \mathbf{H}_{j, n}(\omega) \mathbf{S}_{n}(\omega) \\
& \mathbf{X}_{i_{n}}(\omega)=c_{i_{n}, n} \mathbf{H}_{i_{n}, n}(\omega) \mathbf{S}_{n}(\omega)
\end{aligned}
$$

where the same notation as in (14) is used. From (18), the following can be obtained

$$
\mathbf{X}_{j}(\omega)=\sum_{n \in \mathcal{S}_{j}} \frac{c_{j, n} \mathbf{H}_{j, n}(\omega)}{c_{i_{n}, n} \mathbf{H}_{i_{n}, n}(\omega)} \mathbf{X}_{i, n}(\omega)
$$


Comparing (지) with the single-source equivalent in (14) it turns out that $\left|\mathcal{S}_{j}\right|$ different filters, with frequency responses $\left\{\frac{c_{j, n} \mathbf{H}_{j, n}(\omega)}{c_{i_{n}, n} \mathbf{H}_{i_{n}, n}(\omega)}\right\}_{n \in \mathcal{S}_{j}}$, have to be learnt using the NLMS approach. Thus, $\left|\mathcal{S}_{j}\right|$ different adaptive filters will be learnt using NLMS via the following updating steps:

Step 1: Evaluate the filter output $\hat{x}_{j}(t)$

$$
\hat{x}_{j}(t)=\sum_{n \in \mathcal{S}_{j}} \mathbf{u}_{i_{n}}^{T}(t) \cdot \hat{\mathbf{w}}_{i_{n}, j}(t)
$$

where $\mathbf{u}_{i_{n}}(t):=\left[x_{i_{n}}(t), x_{i_{n}}(t-1), \cdots, x_{i_{n}}(t-\bar{L}+1)\right]^{T}$ corresponds to the input signals from head sensor $i_{n}$ in single-source cluster $\hat{\mathcal{M}}_{n}$, and $\hat{\mathbf{w}}_{i_{n}, j}(t):=\left[w_{i_{n}, j, t}(0)\right.$, $\left.w_{i_{n}, j, t}(1), \ldots, w_{i_{n}, j, t}(\bar{L}-1)\right]^{T}$ corresponds to the $\bar{L}$ coefficients of the adaptive filters used to learn the frequency response $\left\{\frac{c_{j, n} \mathbf{H}_{j, n}(\omega)}{c_{i_{n}, n} \mathbf{H}_{i_{n}, n}(\omega)}\right\}$. This step is done for all $n \in \mathcal{S}_{j}$. Step 2: Calculate the error signal $e_{i_{n}, j}(t)$

$$
e_{i_{n}, j}(t)=\hat{x}_{j}(t)-x_{j}(t)
$$

Step 3: Update the filter coefficients $\mathbf{w}_{i_{n}, j}$ as

$$
\hat{\mathbf{w}}_{i_{n}, j}(t+1)=\hat{\mathbf{w}}_{i_{n}, j}(t)+\mu \frac{e_{i_{n}, j}(t) \mathbf{u}_{i, n}(t)}{\zeta+\mathbf{u}_{i_{n}}^{T}(t) \mathbf{u}_{i_{n}}(t)} .
$$

The coefficients $\hat{\mathbf{w}}_{i_{n}, j}(t+1)$ are iteratively updated until the update difference $\| \hat{\mathbf{w}}_{i_{n}, j}(t)-$ $\hat{\mathbf{w}}_{i_{n}, j}(t-1) \|^{2}$ drops below a desired threshold. In fact, the three updating steps presented earlier could be applied periodically in a setting where the channel coefficients $h_{j, m, t}(\tau)$ are slowly-varying with time.

\subsection{FC Field Reconstruction}

During the operation stage of the proposed scheme, the FC is responsible for reconstructing all sensor measurements using only i) the measurements of the head sensors; and ii) the filter coefficients determined in Sec. 4.4. First, the reconstruction of the measurements of a sensor belonging to a single-source cluster $\hat{\mathcal{M}}_{m}$ is considered at the FC. Let sensor $i_{m}$ denote the head sensor in cluster $\hat{\mathcal{M}}_{m}$ with measurements $x_{i_{m}}(t)$. ${ }_{435}$ Sensor $j$ measurements are reconstructed using the filter $\hat{\mathbf{w}}_{i_{m}, j}$ obtained using NLMS in Sec. 4.4.] as

$$
\hat{x}_{j}(t)=\mathbf{u}_{i_{m}}^{T}(t) \cdot \hat{\mathbf{w}}_{i_{m}, j},
$$


where $\mathbf{u}_{i_{m}}$ contains the head sensor's measurements [cf. Sec. 4.4.J]. If sensor $j$ belongs to a multi-source cluster $\hat{\mathcal{M}}_{m}$, then the FC can reconstruct its measurements readily using the filters $\hat{\mathbf{w}}_{i_{n}, j}$, obtained in Sec. 4.4.2, as follows

$$
\hat{x}_{j}(t)=\sum_{n \in \mathcal{S}_{j}} \mathbf{u}_{i_{n}}^{T}(t) \cdot \hat{\mathbf{w}}_{i_{n}, j},
$$

where $\mathbf{u}_{i_{n}}(t)$ corresponds to the sensor measurements obtained at head sensor $i_{n}$ in neighboring single-source cluster $\hat{\mathcal{M}}_{n}$ [cf. Sec. 4.4.2]

\section{Communication and Computational Costs}

In a setting with dense sensor deployment, the communication cost is an important figure of merit to quantify the efficacy of the algorithm. The proposed framework introduces a significant reduction in the communication cost with respect to a setting where all sensors have to transmit their measurements to the FC every time they sense new information. Specifically, consider a setting with $M$ uncorrelated sources (number of single-source clusters) impacting the $N+T$ measurements acquired across the $p$ sensors, where $N$ corresponds to the number of the training data and $T$ denotes the number of measurements obtained during the operational phase of the algorithm. The proposed scheme entails the transmission of $p N+M T$ scalars. In a standard approach, where all sensors transmit to the $\mathrm{FC}$, the communication cost will be $p(N+T)$ scalars. The reduction in the communication cost takes place during the operation stage of the proposed scheme, the reason is that in practical settings the number of sensors $p$ is much larger than the number of sources $M$, especially when the network consists of densely populated sensing units.

During the training stage of the algorithm, the computational complexity at the FC is $\mathcal{O}\left(N p^{2}\right)$ dominated by PCA. During the operational stage, the reconstruction formulas in (24) and (25), reveal that the computational complexity at the FC is of the order of $\mathcal{O}\left(\bar{L} \cdot T \cdot \sum_{m=1}^{M}\left(\left|\hat{\mathcal{M}}_{m}\right|-1\right)\right)$.

The scalability of the algorithm to handle data from large number of sensors is another important aspect in practical deployments. Employing S-CCA and PCA for a large population of sensors may be challenging. Thus, from the perspective of a practical implementation, the entire field can be split in multiple, physically separated subfields which can be treated separately as described earlier in Secs. 4.1, 4.2 and 
42.2. In each of these subfields the proposed framework is utilized to identify their respective clusters. Then, the merging approach put forth in Sec. 4.2 .2 can be applied in clusters obtained in neighboring subfields.

It is also worth noticing that when the source distribution changes, i.e., moving sources, then the desired clustering pattern along with the filters should also change. Thus, the process of learning and reconstructing the monitored field can be repeated as needed to readjust to changes on the monitored field.

\section{Numerical Tests}

The proposed framework is tested here in terms of clustering efficiency, field reconstruction quality and communication efficiency. To this end, $p$ sensors are deployed within a 2-D field occupying the normalized region $[0,1] \times[0,1]$. The field sources evolve in time according to a first-order autoregressive model (AR-1), i.e., $s_{m}(t)=F_{m} \cdot s_{m}(t-1)+u_{m}(t)$, for $m=1, \ldots, M$ where $F_{m}$ is the autoregressive coefficient which is selected such that $\left|F_{m}\right| \leq 1$, and $u_{m}(t)$ corresponds to zero-mean white perturbation noise with variance 0.1 .

The propagation coefficients for the channels $\left\{h_{j, m}(l)\right\}_{j=1, l=1, m=1}^{p, L, M}$ in (II) are Gaussian distributed under the constraint that their energy is equal to one, i.e., $\left\{\sum_{\tau=0}^{L-1} h_{j, m}^{2}\right.$ $(\tau)=1\}$, where $L=3$. Furthermore, the memory length, namely $f$ in (I) , is set as $f=1$. The sparsity-controlling coefficients, saying $\left\{\lambda_{D, \rho}, \lambda_{E, \rho}\right\}_{\rho=1}^{M}$, are chosen through the $\lambda$-selection scheme in Sec. III-C of [4]. The NLMS-based adaptive filter is considered to have $\bar{L}=20$ taps and the NLMS step-size is set to $\mu=0.01$. Furthermore, the sensing noise variance is set such that the signal-to-noise-ratio (SNR) in the numerical tests is $13 \mathrm{~dB}$. Note that all the simulation graphs below are obtained after averaging over 100 independent Monte Carlo runs. In this section, we apply our proposed algorithm in Sec. 田 to the following three scenarios:

S1) Non-overlapping case: Three uncorrelated sources $s_{1}(t), s_{2}(t)$ and $s_{3}(t)$ are considered to be at positions $[0.3,0.4],[0.8,0.8]$, and $[0.8,0.2]$, respectively. $p=30$ sensors are considered here, and each source is sensed by 10 sensors. Each sensor acquires information about one source only, since sources do not overlap here. 
S2) Overlapping case 1: $p=15$ sensors are deployed in the field in which $M=2$ uncorrelated sources are present. Each source is sensed by 5 single-source sensors, the remaining 5 sensors observe both sources.

S3) Overlapping case 2: There are $p=30$ sensors and $M=4$ sources in the field, see. Fig. [. In detail, 4 sensors (denoted by the purple dots), 4 sensors (red dots), 4 sensors (pink dots), and 3 sensors (blue dots) observe source $s_{1}(t), s_{2}(t), s_{3}(t)$, and $s_{4}(t)$, respectively. Moreover, 3 sensors (the green dots) acquire information about source $s_{1}(t)$ and $s_{2}(t), 4$ sensors (yellow dots) are affected by both sources $s_{3}(t)$ and $s_{4}(t)$, while the remaining sensors (black dots) are far away from any of the four sources and they only sense noise.

\subsection{Clustering Performance}

During the training phase, the number of sources needs to be determined and subse- 
S-CCA performs better in terms of clustering the sensor data and less iterations are required to reallocate sensors that are wrongly clustered. The probability of correct clustering via S-CCA is also studied in Figs. 1,6 and $\square$ versus the number of training data samples available per sensor. Note that the different probability curves are plotted for a different number of S-CCA/PCA iterations to demonstrate how the scheme in Sec. 4.2 .2 gradually improves the clustering accuracy. Figs. [, 6 and $\square$ depict the probability curves for scenarios S1, S2 and S3, respectively. It can be seen clearly in all cases that as the number of iterations increases the probability also increases and eventually reaches one after a certain number of iterations depending on the setting. Note also that the probability increases with the number of training data. Further, the improvement in probability is substantial for the initial iterations, while it declines as more and more iterations are applied and perfect clustering is reached.

\subsection{Signal Reconstruction}

The average reconstruction MSE at the FC is studied here versus the number of training data available. In detail, the performance metric utilized here is the normalized average recovery mean-square error defined as

$$
M S E_{n}=\frac{1}{p} \sum_{j=1}^{p}\left[\frac{\sum_{t=1}^{T}\left(\hat{x}_{j}(t)-x_{j}(t)\right)^{2}}{\sum_{t=1}^{T}\left(x_{j}(t)\right)^{2}}\right],
$$

where the data employed correspond to the data acquired after the training phase during the operational stage. Thus, the normalized average MSE demonstrates how efficient is the proposed algorithm in reconstructing data other than the training data (generalization) that follow the same statistical model. The reconstruction capability of the proposed framework in Sec. 4.4 via NLMS adaptive filtering and relying on the source cluster sensors is depicted in Fig. 8, for scenarios S1, S2 and S3. Fig. 8, corroborates that as the number of training sensor measurements increases the normalized MSE reduces showing the capability of the proposed NLMS-based reconstruction technique in learning the correlation patterns within clusters. Further, the average normalized MSE is similar for all different scenarios considered showing the effectiveness of the proposed scheme irrespective of the setting considered. 


\subsection{Source-Sensor Channel Variation and Communication Efficiency}

The adaptivity of the NLMS's reconstruction ability is tested in a setting where the source-sensor channel coefficients change at a given time. The test setting involves $p=10$ sensors that observe the same source. The channel coefficients $\left\{h_{j}(\ell)\right\}_{j=1, \ell=1}^{10,3}$ are modified at time instant $t=2000$, and sensor 1 is used as the head sensor whose measurements will be used as a reference signal when applying NLMS to reconstruct the measurements corresponding to the remaining 9 sensors. Fig. $Q$ depicts the relative error versus time $t$. The relative reconstruction MSE is evaluated here as

$$
e_{M S E}(t):=\frac{\sum_{j=2}^{10}\left(\hat{x}_{j}(t)-x_{j}(t)\right)^{2}}{\frac{1}{4000} \sum_{t=0}^{3999} \sum_{j=2}^{10}\left(x_{j}(t)\right)^{2}} .
$$

Fig. 9 corroborates the adaptability of the proposed scheme in the event of changes in the channel coefficients. Clearly, when the source-sensor channel coefficients change, the NMLS approach, after an overshoot in the relative MSE at $t=2000$ is able to eventually recover and reconstruct all the sensor measurements accurately . Finally, Fig. 10 illustrates the communication cost in terms of the number of scalars transmitted from the sensors to the FC versus time. The communication cost is depicted for the three different scenarios $\mathrm{S} 1, \mathrm{~S} 2$ and $\mathrm{S} 3$. The training phase lasts up to $t=5000$ where all sensors acquire $N=5000$ measurements used as training data, while the remaining $T=5000$ correspond to the measurements acquired during the operational stage. Notice that during the training phase all sensors transmit to the FC so there is no reduction in communication cost during this period. However, during the operational phase where sensor clustering has been performed and only the head sensors of each cluster transmit information to the FC the reduction in communication cost is substantial compared to a setting where all sensors keep transmitting their measurements to the FC. Fig. 10 depicts the substantial reduction in the number of scalars that need to be communicated from the head sensors to the FC during the operational phase of the novel framework.

\section{Conclusion}

A novel framework was put forth for sensor clustering and communication efficient field reconstruction. Norm-one regularized canonical correlations were combined with 
principal component analysis and moving-average filtering to successfully cluster sensors according to their information content, as the number of training data and movingaverage filter length goes to infinity. Utilizing data only from pertinent head sensors in each cluster, the FC reconstructs all sensors' measurements using effective normalized least mean squares techniques, reducing substantially the communication cost. Numerical experiments demonstrated the capability of the proposed novel approach in field reconstruction.

\section{Appendix A. Impact of Moving Average Filter}

Consider the measurement acquired at sensor $j$ at time instant $t$

$$
x_{j}(t)=\sum_{m \in \mathcal{S}_{j}} c_{j, m} \sum_{\tau=0}^{L-1} h_{j, m}(\tau) s_{m}(t-\tau)+w_{j}(t)
$$

where $\mathcal{S}_{j}$ corresponds to the set that contains the source indices observed by sensor $j$.

The MA filtering applied here boils down to form a running sample-average of $P$ consecutive measurements at time instant $t$, i.e.,

$$
\begin{aligned}
\bar{x}_{j}(t) & =\frac{1}{P} \sum_{\ell=t}^{t-P+1}\left[\sum_{m \in \mathcal{S}_{j}} c_{j, m} \sum_{\tau=0}^{L-1} h_{j, m}(\tau) s_{m}(\ell-\tau)+w_{j}(\ell)\right] \\
& \sum_{m \in \mathcal{S}_{j}} c_{j, m} \sum_{\tau=0}^{L-1} h_{j, m}(\tau)\left[P^{-1} \sum_{\ell=t}^{t-P+1} s_{m}(\ell-\tau)\right]+\frac{1}{P} \sum_{\ell=t}^{t-P+1} w_{j}(\ell) \\
= & \sum_{m \in \mathcal{S}_{j}} c_{j, m} \sum_{\tau=0}^{L-1} h_{j, m}(\tau) \bar{s}_{m}(t-\tau)+\bar{w}_{j}(t),
\end{aligned}
$$

where $\bar{s}_{m}(t-\tau):=P^{-1} \sum_{\ell=t}^{t-P+1} s_{m}(\ell-\tau)$ and $\bar{w}_{j}(\ell)$ corresponds to the averaged noise term in (29).

Note that if the length of the MA filter $P$ is selected sufficiently large compared to

L, while the source signals have a time-invariant ensemble average (e.g., wide sense stationary processes) then in that case it holds that

$$
\bar{s}_{m}(t) \approx \bar{s}_{m}(t-1) \approx \ldots \approx \bar{s}_{m}(t-L+1),
$$

due to the averaging effect of a sufficiently large number of sensor measurements. Then, after utilizing (BतI) in the third equation in (ㅁ) we obtain

$$
\bar{x}_{j}(t) \approx \sum_{m \in \mathcal{S}_{j}} c_{j, m} \sum_{\tau=0}^{L-1} h_{j, m}(\tau) \bar{s}_{m}(t)+\bar{w}_{j}(t)=\sum_{m \in \mathcal{S}_{j}} \bar{H}_{j, m} \bar{s}_{m}(t)+\bar{w}_{j}(t),
$$


where $\bar{H}_{j, m}:=c_{j, m} \sum_{\tau=0}^{L-1} h_{j, m}(\tau)$.

From (BII) it follows readily that the noise $\bar{w}_{j}(t)$ has zero-mean and variance $\sigma_{w, j}^{2} / P$. Thus, for sufficiently large $P$ the noise can be made negligible and virtually be ignored. Next, it is demonstrated how the MA measurements can be utilized to obtain an accurate estimate for the number of sources present in the monitored field. Stacking all the MA measurements in (BII) in a vector $\overline{\mathbf{x}}_{t}$ we obtain

$$
\overline{\mathbf{x}}_{t}:=\left[\bar{x}_{1}(t) \ldots \bar{x}_{p}(t)\right]^{T}=\sum_{m=1}^{M} \overline{\mathbf{h}}_{m} \bar{s}_{m}(t),
$$

590

595

where $\overline{\bar{s}}_{m}:=\bar{N}^{-1} \sum_{t=1}^{\bar{N}} \bar{s}_{m}(t)$ and $\overline{\bar{s}}_{m^{\prime}}:=\bar{N}^{-1} \sum_{t=1}^{\bar{N}} \bar{s}_{m^{\prime}}(t)$. For sufficiently large number of training data $\bar{N}$ and since the sources are uncorrelated it turns out from Law of Large Numbers[14]

$$
\bar{N}^{-1} \sum_{t=1}^{\bar{N}}\left[\bar{s}_{m}(t)-\overline{\bar{s}}_{m}\right]\left[\bar{s}_{m^{\prime}}(t)-\overline{\bar{s}}_{m^{\prime}}\right] \approx \sigma_{m}^{2} \delta\left(m-m^{\prime}\right)
$$

with $\delta\left(m-m^{\prime}\right)$ denoting the Kronecker delta function and $\sigma_{m}^{2}$ denotes the variance of source $s_{m}(t)$. Thus, the MA data covariance matrix in (B3]) can be written as

$$
\hat{\boldsymbol{\Sigma}}_{\bar{x}} \approx \sum_{m=1}^{M} \sigma_{m}^{2} \overline{\mathbf{h}}_{m} \overline{\mathbf{h}}_{m}^{T} .
$$

As long as the column vectors $\overline{\mathbf{h}}_{m}$ are linearly independent it is easily seen that for sufficiently large number of training data $N$, and MA filtering length $P$ the number of nonzero eigenvalues of the MA data covariance matrix estimate $\hat{\boldsymbol{\Sigma}}_{\bar{x}}$ is equal to the number of sources $M$. Note that for the random Gaussian channel coefficients considered in Sec. [3, the vectors $\left\{\overline{\mathbf{h}}_{m}\right\}_{m=1}^{M}$ are linearly independent. 


\section{Appendix B. Correct Clustering Proof}

Consider sensor $j$ in which after applying the MA filter to its measurements following (II), and utilizing the result from (29) it follows

$$
\bar{x}_{j}(t)=\sum_{m \in \mathcal{S}_{j}} c_{j, m} \sum_{\tau=0}^{L-1} h_{j, m}(\tau)\left[P^{-1} \sum_{p=t}^{t-P+1} s_{m}(p-\tau)\right]+\frac{1}{P} \sum_{p=t}^{t-P+1} w_{j}(p)
$$

where $\mathcal{S}_{j}$ corresponds to the set of sources that are affecting the measurements of sensor $j$. When the number of measurements $P$ is selected sufficiently large, the noise variance in (B7) can be made arbitrarily small as stated in Apdx. A. Thus, for sufficiently large $P$ the MA measurements in (B7) can be assumed approximately to be noise free which further implies that the sensor measurements are scaled versions of the modified source signal $\bar{s}_{m}(t)$. Since the modified source signal $\bar{s}_{m}(t)$ is formed by adding a set of $P$ samples of the original source signal $s_{m}(t)$, and the original source signals are uncorrelated then the modified source signals are also uncorrelated.

It is demonstrated how the recursive interplay between PCA and CCA in Sec. 4.2 .2 ensures the correct clustering of the sensors according to their source content for sufficiently large MA filter length $P$ and number of training data $N$. S-CCA at first is applied across all the sensors' MA measurements which are stacked in vectors $\overline{\mathbf{x}}(t)$ for $t=1, \ldots, \bar{N}$ and used to form the MA data covariance matrix $\Sigma_{x}^{0}$ using (1D2) and all measurements in $\overline{\mathbf{x}}(t)$. For sufficiently large $P$, the eigenvalues of $\Sigma_{x}^{0}$ corresponding to the sources will be significantly larger in magnitude than the eigenvalues corresponding to noise which will have negligible magnitude. Thus, by selecting a proper threshold (dependent on the value of $P$ ) the source-related eigenvalues can be identified, and their cardinality will correspond to the actual number of sources $M$. Then, S-CCA 625 can be employed to identify the $M$ different clusters $\hat{\mathcal{M}}_{m^{1}}^{1}$ for $m^{1}=1, \ldots, M$. For each of these clusters the corresponding sensor measurements are stacked in vectors $\overline{\mathbf{x}}_{1}^{1}(t), \overline{\mathbf{x}}_{2}^{1}(t), \ldots, \overline{\mathbf{x}}_{M}^{1}(t)$ for $t=1, \ldots, \bar{N}$ respectively, where the subscript indicates the cluster index and the superscript the iteration index. The aforementioned vectors are used in the same way as during the first iteration to form via sample-averaging the covariance matrices $\Sigma_{x, m^{1}}^{1}$ in (ㄴ2). After PCA is applied to each $\Sigma_{x, m^{1}}^{1}$, the number 
of sources contained in the $m$ th cluster measurements $\overline{\mathbf{x}}_{m}^{1}(t)$ can be determined as delineated earlier. The alternating interplay between S-CCA and PCA is continued until all resulting clusters either contain information about only one source, or have a single measurement that may contain information about multiple sources.

During the merging process, when PCA is applied for sufficiently large $P$ and $N$ as described in Sec. 4.2.2 then after a finite number of iterations, which will not exceed the number of sensors $p$ (worst case scenario where all clusters contain a single-sensor measurements), this process will result $M$ clusters that contain the measurements of sensors observing a single common source. If there are sensors that sense more than one sources, then there will also be clusters that contain measurements which have information about these sources. The source content of these multi-source clusters will be identified by employing the PCA process described in Sec. 4.2 .2 which will determine the single-source clusters that share the same source content with the multisource cluster.

\section{References}

[1] A. Avokh and G. Mirjalily, "Dynamic Balanced Spanning Tree (DBST) for Data Aggregation in Wireless Sensor Networks," Proc. of International Symposium on Telecommunication (IST), pp. 391-396, 2010.

[2] J. M. Bruce and P. L. Dragotti, "Reconstructing Diffusion Fields Sampled with a Network of Arbitrarily Distributed Sensor," Proc. of 22nd European Signal Processing Conference (EUSIPCO), Lisbon, Portugal, Sep. 2014.

[3] D. R. Brillinger, Time Series: Data Analysis and Theory. Expanded Edition, Holden Day, 1981.

[4] J. Chen and I. D. Schizas, "Online Distributed Sparsity-Aware Canonical Correlation Analysis," IEEE Trans. on Signal Processing, vol. 64, no. 3, pp. 688-703, 2016.

[5] J. Chen and I. D. Schizas, "Regularized Canonical Correlations for Sensor Data Clustering," IEEE Int. Conf. on Acoustics, Speech and Signal Processing (ICASSP), Brisbane, Australia, April 2015.

[6] J. Chen, I. D. Schizas, "Distributed information-based cluster- 
ing of heterogeneous sensor data," Signal Processing, available at http://dx.doi.org/10.1016/j.sigpro.2015.12.017, 2016.

[7] M. M. Chiang and B. Mirkin, "Intelligent Choice of the Number of Clusters in kMeans Clustering: An Experimental Study with Different Cluster Spreads," Journal of Classification, vol. 27, no. 3, pp. 3-40, 2010.

[8] J. H. Cui, J. Kong, M. Gerla, and S. Zhou, "The Challenges of Building Scalable Mobile Underwater Wireless Sensor Networks for Aquatic Applications," IEEE Networks, no. 20, pp. 12-18, 2006.

[9] I. Dokmanic, J. Ranieri, A. Chebira, and M. Vetterli, "Sensor Networks for Diffusion Fields: Detection of Sources in Space and Time," Proc. of 49th Allerton Conf. Commun., Control, Comput. (Allerton), pp. 1552-1558, Monticello, Sep. 2011.

[10] J. M. Hoem, "A contribution to the statistical theory of linear graduation," Insurance: Mathematics and Economics, vol. 3, no. 1, pp. 117, 1984, doi: 10.1016/0167-6687(84)90014-3.

[11] D. H. Hoang, T. Strufe, Q. Le, P. Bui, T. Pham, N. Thai, T. Le, and E. Schweizer, "Processing and Visualizing Traffic Pollution Data in Hanoi City from a Wireless Sensor Network," Local Computer Networks Workshops (LCN Workshops), pp. 21-24, Sydney, Oct. 2013.

[12] Y. M. Lu, P. L. Dragotti, and M. Vetterli, "Localizing Point Sources in Diffusion Fields from Spatiotemporal Samples," Proc. of the 9th Int. Conf. Sampl. Theory Appl. (SampTa), Singapore, May 2011.

[13] S. Lloyd, "Least Squares Quantization in PCM," IEEE Trans. Inf. Theory, vol. 28, no. 2, pp. 129-137, Mar. 1982.

[14] M. Loeve, Probability Theory 1. Fourth Edition, Springer Verlag, 1977.

[15] D. Mantri, N. R. Prasad and R. Prasad, "BHCDA: Bandwidth efficient Heterogeneity Aware Cluster Based Data Aggregation for Wireless Sensor Network," Proc. of IEEE Intl. Conf. of Advances in Computing, Communications and Informatics (ICACCI), pp. 1064-1069, Aug. 2013.

[16] J. Matthes, L. Groll, and H. B. Keller, "Source Localization by Spatially Distributed Electronic Noses for Advection and Diffusion," IEEE Trans. Signal Processing, vol. 53, no. 5, pp. 1711-1719, May 2005. 
[17] J. Matamoros, F. Fabbri, C. A. Haro, and D. Dardari, "On the Estimation of Randomly Sampled 2D Spatial Fields under Bandwidth Constrains," IEEE Trans. Wireless Communications, pp. 4184- 4192, vol. 10, no. 12, Dec. 2011.

[18] A. Nehorai, B. Porat, and E. Paldi, "Detection and Localization of Vapor-Emitting Sources," IEEE Trans. Signal Processing, vol. 43, no. 1, pp. 243-253, Jan. 1995.

[19] A. Nordio, C. F. Chiasserini, and E. Viterbo, "Bandlimited field reconstruction for wireless sensor networks," Tech Rep., Politecnico di Torino, Jan. 2006.

[20] N. S. Patil and P. R. Patil, "Data Aggregation in Wireless Sensor Network", IEEE Int. Conf. Computational Intelligence and Computing Research, Dec. 2010.

[21] P. Patil and U. Kulkarni, "SVM based Data Redundancy Elimination for Data Aggregation in Wireless Sensor Networks, in processing of Advances in Computing, Communications and Informatics (ICACCI), International Conference IEEE, pp. 1309-1316, Aug. 2013.

[22] G. Ren, V. Maroulas, and I. D. Schizas, "Distributed Spatio-Temporal Association and Tracking of Multiple Targets Using Multiple Sensors", IEEE Transactions on Aerospace and Electronic Systems, vol. 51, no. 4, pp. 25702589, 2015.

[23] J. Ranieri, A. Vincenzi, A. Chebira, D. A. Alonso, and M. Vetterli, "EigenMaps: Algorithms for Optimal Thermal Maps Extraction and Sensor Placement on Multicore Processors," Proc. 49th Design Autom. Conf. (DAC), ACM, pp. 636-641, San Francisco, 2012.

[24] G. Reise, G. Matz, and K. Grochenig, "Distributed Field Reconstruction in Wireless Sensor Networks Based on Hybrid Shift-Invariant Spaces," IEEE Trans. Signal Process. vol. 60, no. 10, pp. 5426-5437, Oct. 2012. 
pp. 2408-2421, May 2012.

[28] S. Sirsikar and S. Anavatti, "Issues of Data Aggregation Methods in Wireless Sensor Network: A Survey," Proc. of 4th International Conference on Advances in Computing, Communication and Control (ICAC), vol. 49, pp. 194-201, 2015.

[29] J. Szurley, A. Bertrand, and M. Moonen, "Distributed Adaptive Node-specific Signal Estimation in Heterogeneous and Mixed-topology Wireless Sensor Networks”, Signal Processing, vol. 117, pp. 44-60, 2015.

[30] F. Sawo, K. Roberts, and U. D. Hanebeck, "Bayesian Estimation of Distributed Phenomena Using Discretized Representations of Partial Differential Equations," Proc. 3rd IEEE Int. Conf. Inf. Control, Autom., Robot. (ICINCO), pp. 16-23, Aug. 2006.

[31] J. Weimer, B. Sinopoli, and B. H. Krogh, "Multiple Source Detection and Localization in Advection-Diffusion Processes Using Wireless Sensor Networks," Proc. 30th IEEE Real-Time Syst. Symp. (RTSS), pp. 333-342, Washington, DC, 2009.

[32] B. Widrow and S. Steam, "Adaptive Signal Processing," 1st Edition, Prentice Hall, Mar. 1985.

[33] Z. Yong, Z. Hui, G. Dongqiang, and W. Zhihua, "Determination of Chemical Point Source Using Distributed Algorithm in Sensors Network," Proc. 24th Chin. Control Decision Conf. (CCDC), pp. 3373-3377, May, 2012.

[34] O. Younis, M. Krunz, and S. Ramasubramanian, "Node clustering in wireless sensor networks: recent developments and deployment challenges," IEEE Networks, no. 20, pp. 20-25, 2006.

[35] L. Yu, N. Wang, X. Meng, “ Real-Time Forest Fire Detection With Wireless Sensor Networks," International Conference on Wireless Communications, Networking and Mobile Computing Proceedings, pp. 1214-1217, Wuhan, China, 2005.

[36] M. Zheng, M. Goldenbaum, S. Stanczak, and H. Yu, "Fast Average Consensus in Clustered Wireless Sensor Networks by Superposition Gossiping," IEEE Wireless Communications and Networking Conference: Mobile and Wireless Networks, pp. 1982-1987, Paris, France, April, 2012. 

$\% \%$ For simplicity, we set $\lambda_{D, \rho}=\lambda_{E, \rho}=\lambda_{\rho}$ for $\rho=1, \cdots, M$. Let $\left\{\lambda_{\rho}^{\max }\right\}_{\rho=1}^{M}$ denote the smallest values of $\left\{\lambda_{\rho}\right\}_{\rho=1}^{M}$ that result $\hat{\mathbf{D}}_{\rho}$ : and $\hat{\mathbf{E}}_{\rho}$ : to be zeros, which are estimated by $\left\{\hat{\lambda}_{\rho}^{m}\right\}_{\rho=1}^{M}$. Denote the zero column index set of $\mathbf{D}$ or $\mathbf{E}$ as $\mathcal{C} . \% \%$

3: Form two data sequences $\left\{\chi_{t}, \boldsymbol{\psi}_{t}\right\}$ via (Б).

$\% \%$ Part 1: Estimate $\lambda_{\rho}^{\max } \% \%$

4: Initialize $\left\{\hat{\lambda}_{\rho}^{m}>0\right\}_{\rho=1}^{M}$ randomly.

5: while(true)

Find $\hat{\mathbf{D}}$ and $\hat{\mathbf{E}}$ via $\mathbf{A l g}$. 1 using set $\hat{\lambda}_{\rho}^{m}$.

if $\hat{\mathbf{D}}_{\rho:} \neq \mathbf{0}\left(\right.$ or $\left.\hat{\mathbf{E}}_{\rho:} \neq \mathbf{0}\right)$

$\hat{\lambda}_{\rho}^{m} \leftarrow 1.5 \hat{\lambda}_{\rho}^{m}$

else if $\hat{\mathbf{D}}_{\rho:}=\mathbf{0}\left(\right.$ or $\left.\hat{\mathbf{E}}_{\rho:}=\mathbf{0}\right)$

$\hat{\lambda}_{\rho}^{m} \leftarrow 0.75 \hat{\lambda}_{\rho}^{m}$

Find $\hat{\mathbf{D}}$ and $\hat{\mathbf{E}}$ via Alg. 1 using $\hat{\lambda}_{\rho}^{m}$.

if $\hat{\mathbf{D}}_{\rho:}=\mathbf{0}\left(\right.$ or $\left.\hat{\mathbf{E}}_{\rho:}=\mathbf{0}\right)$

$\hat{\lambda}_{\rho}^{m} \leftarrow 0.75 \hat{\lambda}_{\rho}^{m}$

else if $\hat{\mathbf{D}}_{\rho:} \neq \mathbf{0}\left(\right.$ or $\left.\hat{\mathbf{E}}_{\rho:} \neq \mathbf{0}\right)$

Break while

end if

end if

end while

$\% \%$ Part 2: Estimate zero column index of $\mathbf{D}$ (and $\mathbf{E}) . \%$

6: Find zero column index set $\mathcal{C}_{1}$ of $\mathbf{D}$ and $\mathbf{E}$ estimates via $\mathbf{A l g}$. $\mathbf{1}$ using $0.1 \hat{\lambda}_{\rho}^{m}$.

7: Find zero column index set $\mathcal{C}_{2}$ of $\mathbf{D}$ and $\mathbf{E}$ estimates via $\mathbf{A l g}$. 1 using $0.01 \hat{\lambda}_{\rho}^{m}$.

8: $\mathcal{C} \leftarrow \mathcal{C}_{1} \cap \mathcal{C}_{2}$

9: $\lambda_{\rho} \leftarrow \hat{\lambda}_{\rho}^{m}$

10: while(true)

$\lambda_{\rho} \leftarrow 0.95 \lambda_{\rho}$

Find $\hat{\mathbf{D}}$ and $\hat{\mathbf{E}}$ via $\mathbf{A l g} .1$ using $\lambda_{\rho}$

if resulting zero column index set for $\hat{\mathbf{D}}($ or $\hat{\mathbf{E}})=\mathcal{C}$

Break while 
Algorithm 3 : Clustering via Alternating S-CCA and PCA

1: Input : Sensor measurements $\left\{\overline{\mathbf{x}}_{t}\right\}_{t=1}^{\bar{N}}$

2: Output : Estimate the number of the sources $\hat{M}$ and clusters $\left\{\hat{\mathcal{M}}_{m}\right\}_{m=1}^{\hat{M}}$

$\% \% \operatorname{PCA}\left(\mathbf{p}_{1}\right)$ returns the number of PCs when applying PCA on the sensor measurements from sensor set $\mathbf{p}_{1}$. Run $\left\{\mathbf{O}_{i}\right\}_{i=1}^{p_{2}}=\mathbf{A l g}$. $2(\cdot)$ generating outputs $\left\{\mathbf{O}_{i}\right\}_{i=1}^{p_{2}}$. Vectors $\left\{\overline{\mathbf{x}}_{m^{1}, m^{2}, \cdots, m^{k}}(t)\right\}_{t=1}^{\bar{N}}$ stack the sensor measurements from the corresponding cluster $\hat{\mathcal{M}}_{m^{1}, m^{2}, \cdots, m^{k}} \% \%$

$\% \%$ Part 1: Estimate the number of sources\% $\%$

3: $\hat{M}=\operatorname{PCA}(\{1,2, \cdots, p\})$

$\% \%$ Part 2: Split clusters making sure PCA(·) returns one $\mathrm{PC}$ in each cluster)\%\%

4: $\hat{\mathcal{M}}_{m^{1}}=\operatorname{Alg} . \mathbf{2}\left(\left\{\overline{\mathbf{x}}_{t}\right\}_{t=1}^{\bar{N}}, \hat{M}, p, f\right)$

5: for $k=1,2, \cdots, p$

$\mathrm{c}=0$

for $m^{1}=1, \cdots, \hat{M}$

...

for $m^{k-1}=1, \cdots, \ell(\ell \leq \hat{M})$

$M_{m^{1}, \cdots, m^{k-1}}=\operatorname{PCA}\left(\hat{\mathcal{M}}_{m^{1}, \cdots, m^{k-1}}\right)$

if $M_{m^{1}, \cdots, m^{k-1}}=1$

$\hat{\mathcal{M}}_{m^{1}, \cdots, m^{k-1}, 1} \leftarrow \hat{\mathcal{M}}_{m^{1}, \cdots, m^{k-1}}, c \leftarrow c+1, \hat{\mathcal{M}}_{c} \leftarrow \hat{\mathcal{M}}_{m^{1}, \cdots, m^{k-1}, 1}$

else if

If $M_{m^{1}, \cdots, m^{k-1}}>1$, then,

$t p=$ Alg. $2\left(\left\{\overline{\mathbf{x}}_{m^{1}, m^{2}, \cdots, m^{k-1}}(t)\right\}_{t=1}^{\bar{N}}, M_{m^{1}, \cdots, m^{k-1}},\left|\hat{\mathcal{M}}_{m^{1}, \cdots, m^{k-1}}\right|, f\right)$

$\left\{\hat{\mathcal{M}}_{m^{1}, \cdots, m^{k-1}, m^{k}}\right\}_{m^{k}=1}^{M_{m^{1}, \ldots, m^{k-1}}} \leftarrow t p$

for $m^{k}=1: M_{m^{1}, \cdots, m^{k-1}}$

$c \leftarrow c+1, \hat{\mathcal{M}}_{c} \leftarrow \hat{\mathcal{M}}_{m^{1}, \ldots, m^{k-1}, m^{k}}$

end for

end if

end for

...

end for

if for all $m^{1}, m^{2}, \cdots, m^{k}, M_{m^{1}, \cdots, m^{k}}=1$

Break for

else

Continue 


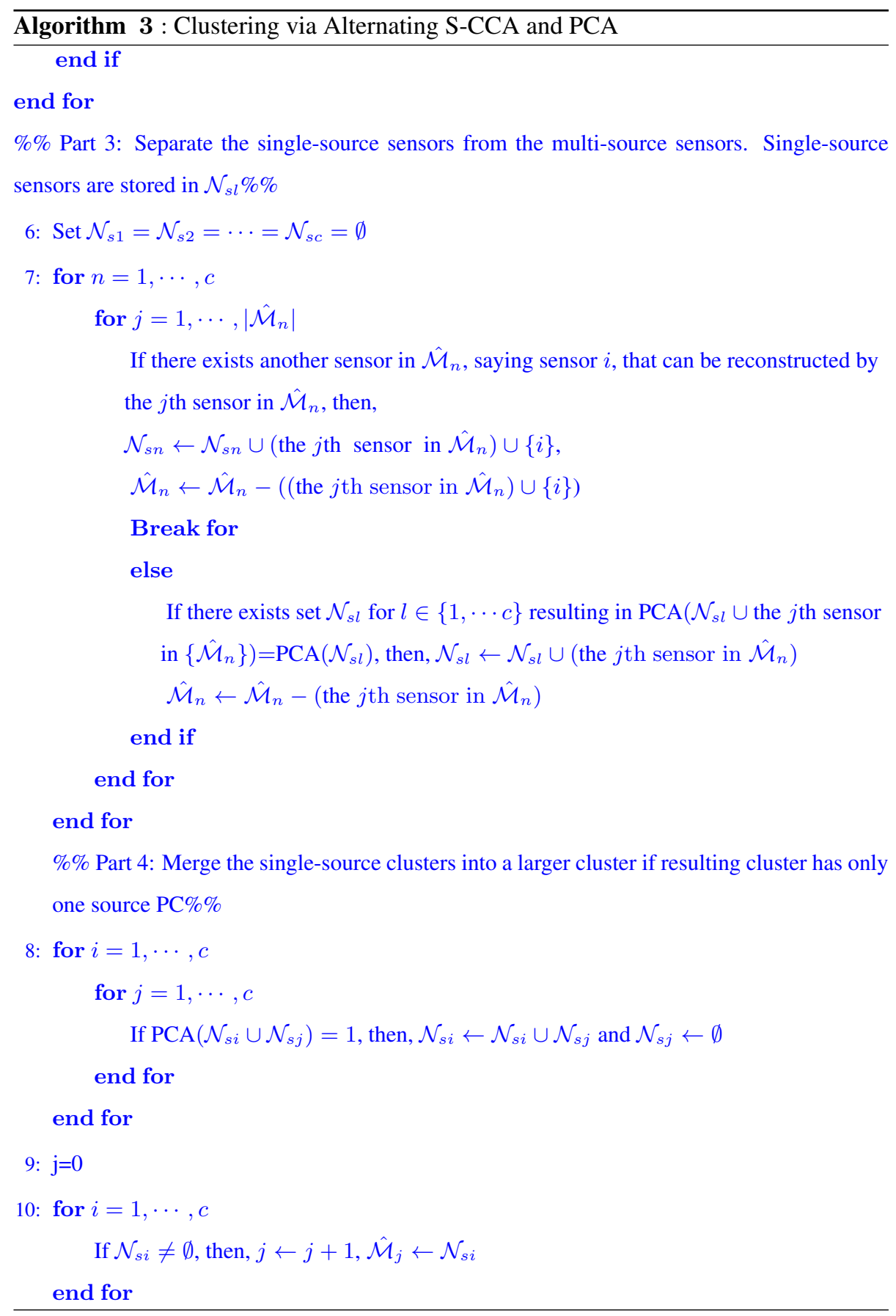




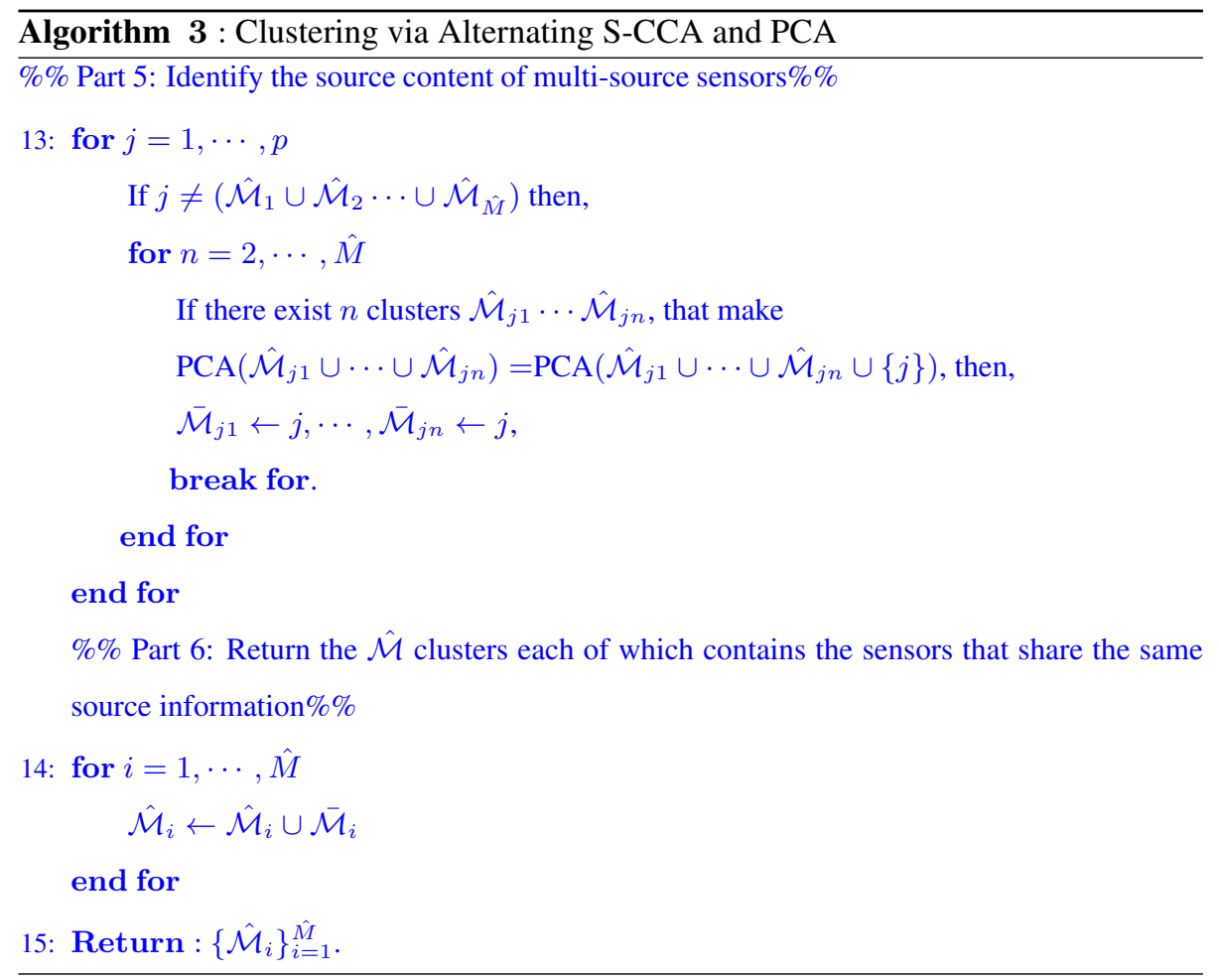




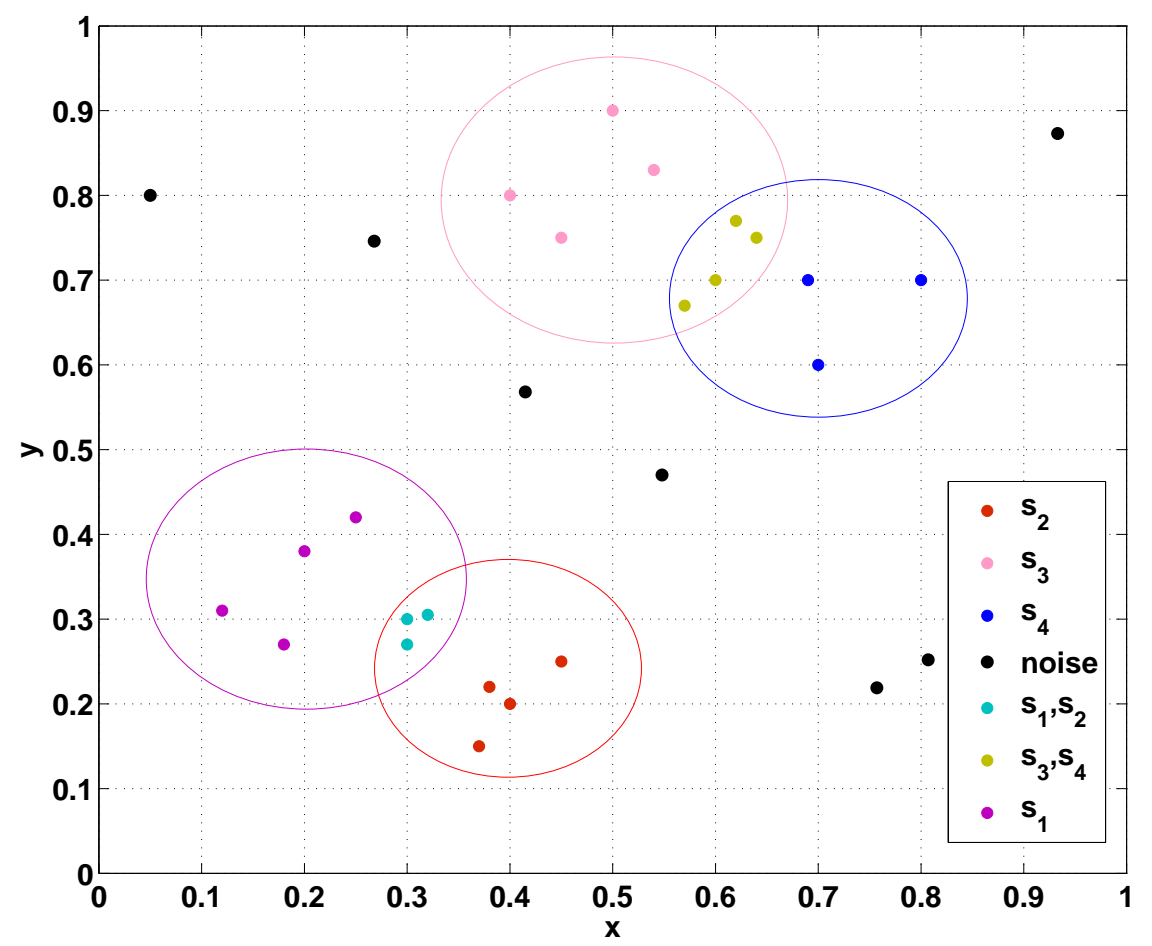

Figure 1: Configuration for overlapping case 2. 
Figure 2: Relative strength of the eigenvalues in the data covariance before (A) and after (B) applying MA filtering for the non-overlapping case. 
Figure 3: Relative strength of the eigenvalues in the data covariance before (A) and after (B) applying MA filtering in overlapping case 1 . 


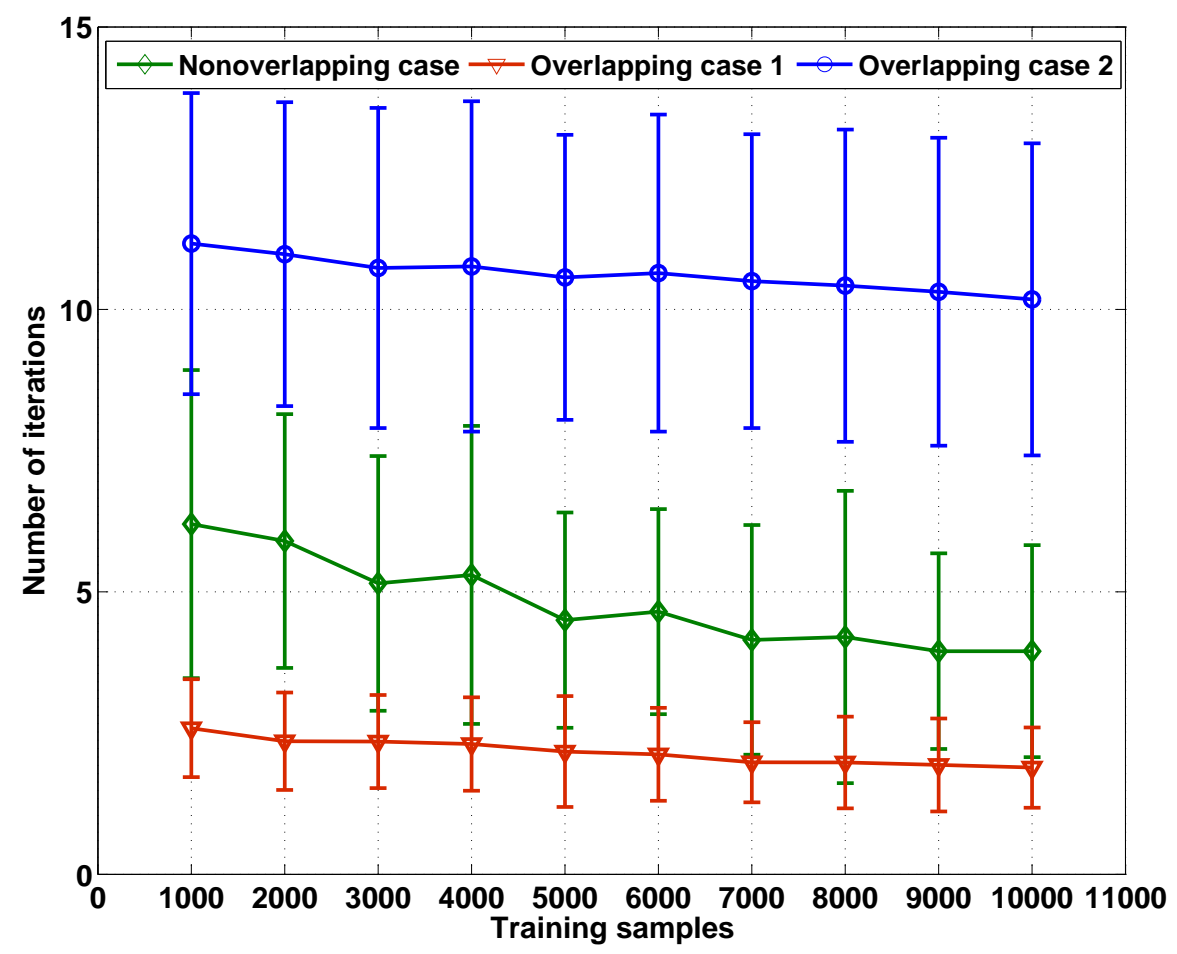

Figure 4: Number of iterations required for perfect clustering vs. number of training samples. 
Figure 5: Probability of correct sensor clustering vs. number of training samples for different number of S-CCA/PCA iterations for scenario S1. 
Figure 6: Probability of correct sensor clustering vs. number of training samples for different number of S-CCA/PCA iterations for scenario S2. 


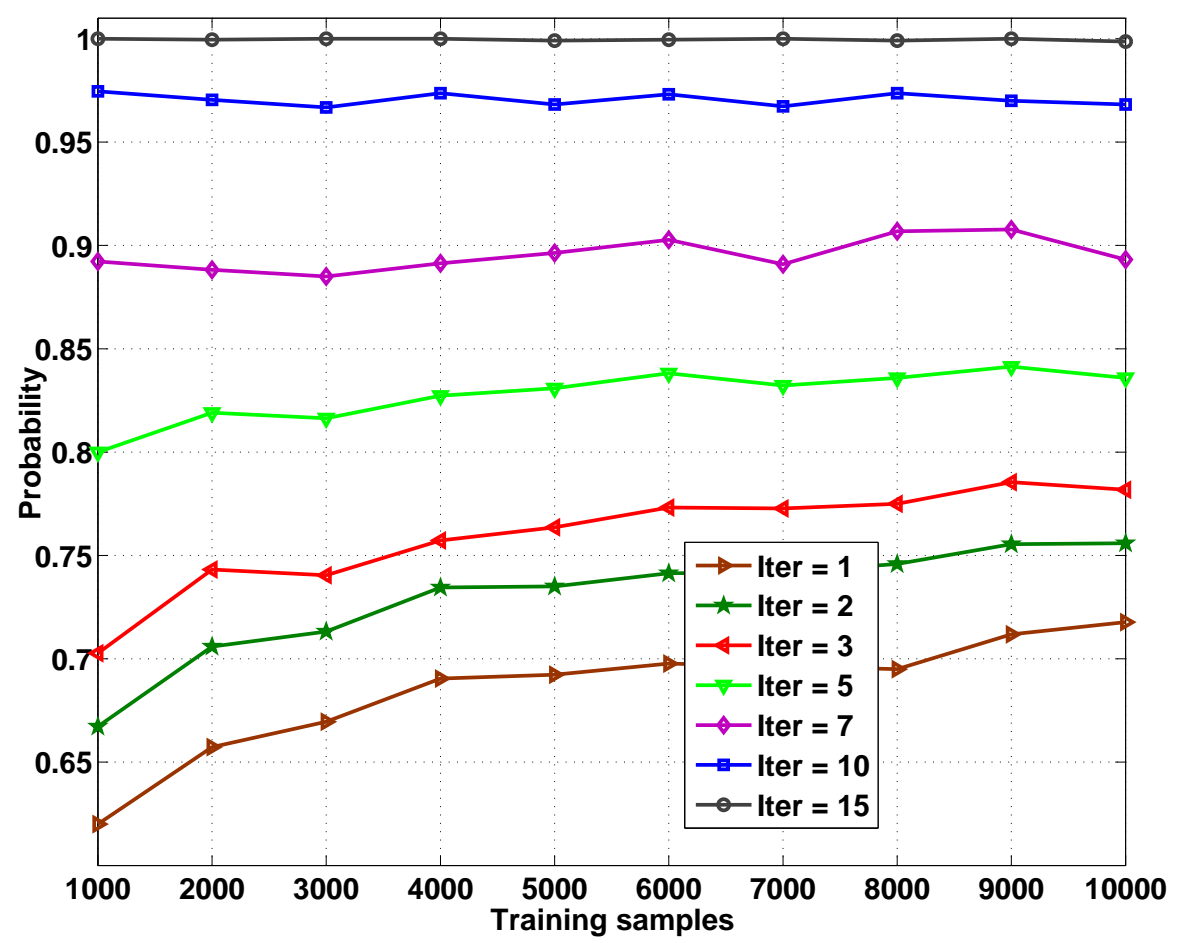

Figure 7: Probability of correct sensor clustering vs. number of training samples for different number of S-CCA/PCA iterations for scenario S3. 


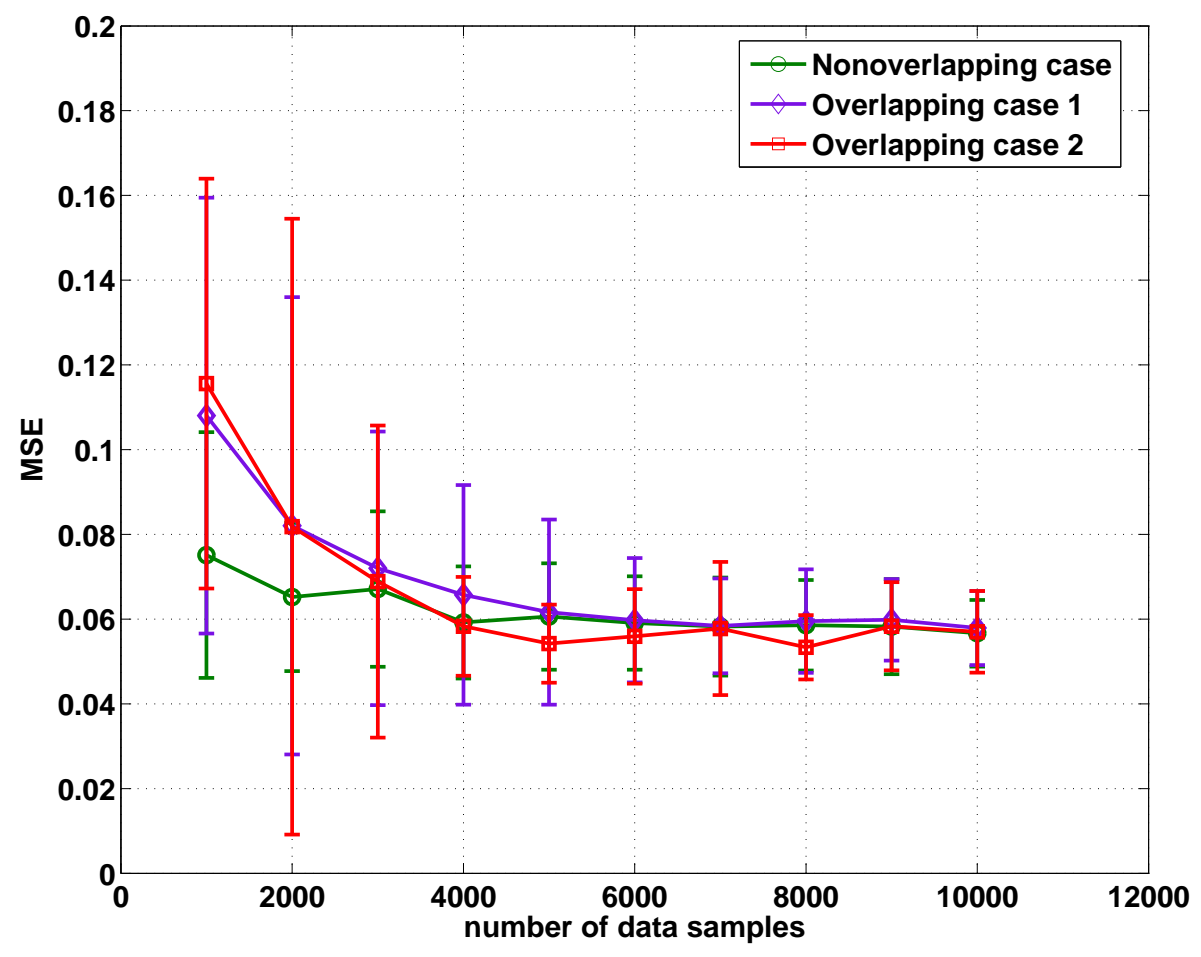

Figure 8: Normalized average reconstruction MSE for scenarios S1,S2 and S3. 


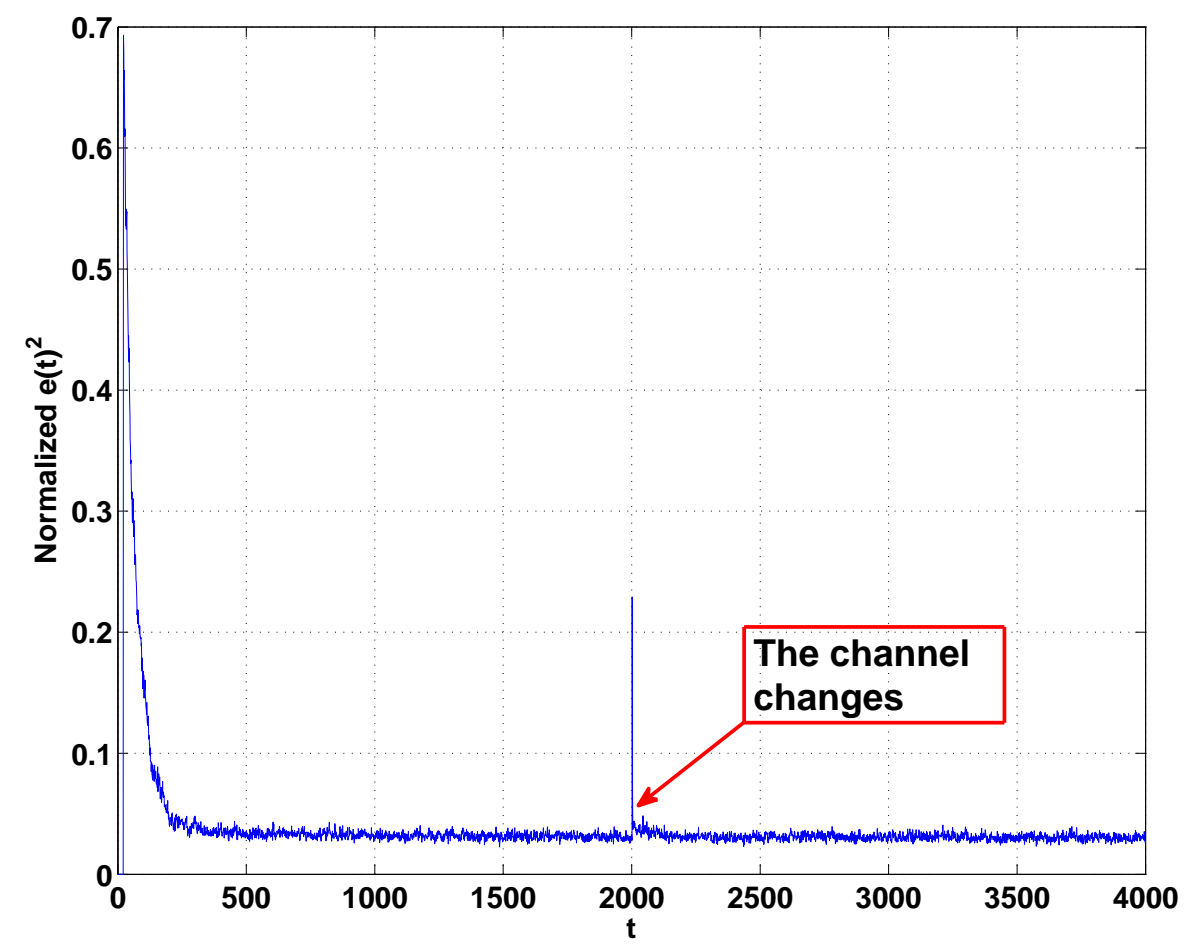

Figure 9: Relative average reconstruction MSE when source-sensor channels change at $t=2000$. 


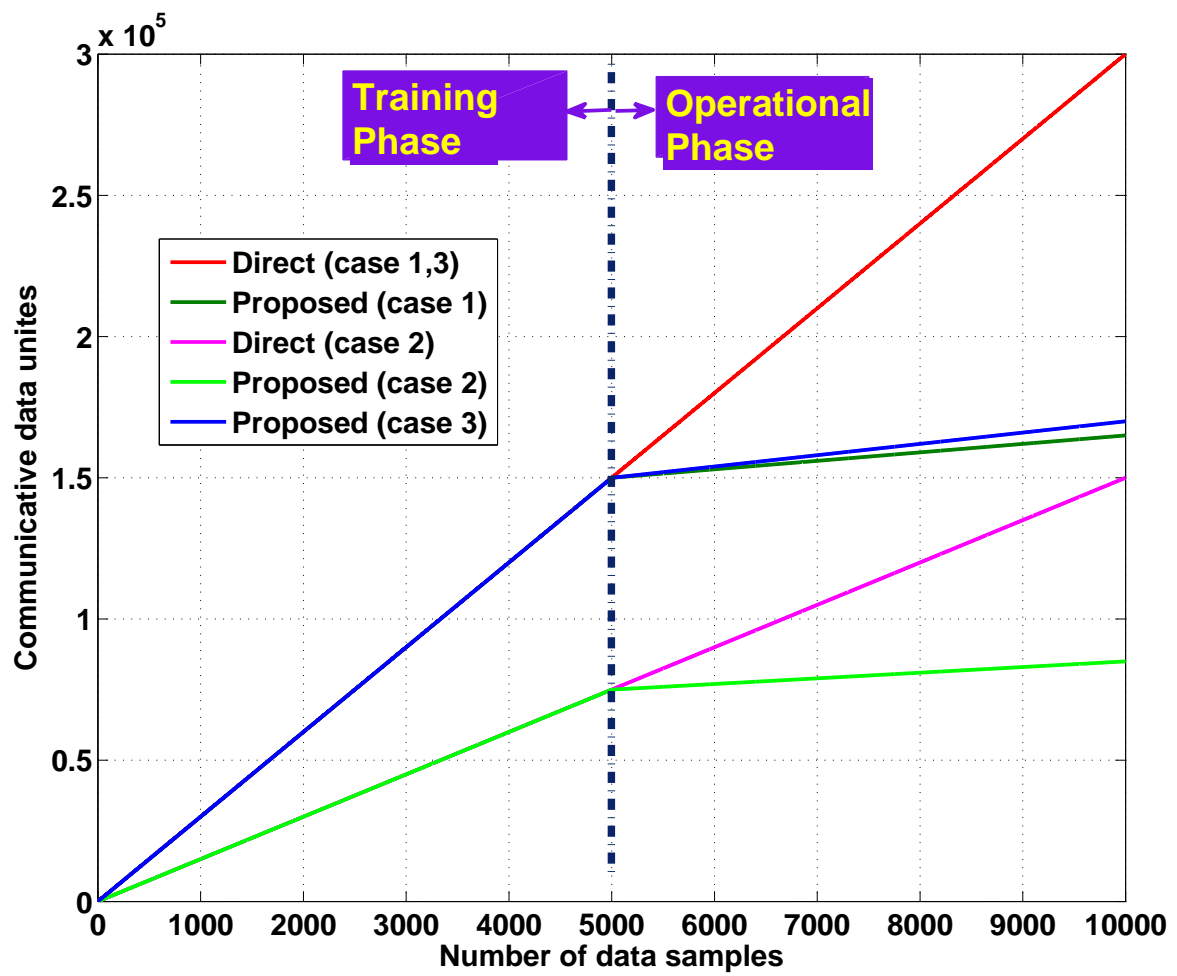

Figure 10: Communication cost versus time for the cases where all sensors, or only the head sensors transmit information to the FC. 